ESAIM: COCV 27 (2021) 106

https://doi.org/10.1051/cocv/2021103
ESAIM: Control, Optimisation and Calculus of Variations

www.esaim-cocv.org

\title{
COST OF NULL CONTROLLABILITY FOR PARABOLIC EQUATIONS WITH VANISHING DIFFUSIVITY AND A TRANSPORT TERM
}

\author{
Jon Asier BÁrCenA-Petisco ${ }^{1,2, *}$ @
}

\begin{abstract}
In this paper we consider the heat equation with Neumann, Robin and mixed boundary conditions (with coefficients on the boundary which depend on the space variable). The main results concern the behaviour of the cost of the null controllability with respect to the diffusivity when the control acts in the interior. First, we prove that if we almost have Dirichlet boundary conditions in the part of the boundary in which the flux of the transport enters, the cost of the controllability decays for a time $T$ sufficiently large. Next, we show some examples of Neumann and mixed boundary conditions in which for any time $T>0$ the cost explodes exponentially as the diffusivity vanishes. Finally, we study the cost of the problem with Neumann boundary conditions when the control is localized in the whole domain.
\end{abstract}

Mathematics Subject Classification. 35B25, 35K05, 93B05, 93C20.

Received April 5, 2021. Accepted November 28, 2021.

\section{INTRODUCTION}

The transport-diffusion equation with vanishing diffusivity is used to describe the dynamics of some physical and biological phenomena. In particular, this is the case of fluid dynamics, as it is explained in Chapter 3 of [1] and the reference therein. Similarly, it is also used to study quantitative biomedical optics, as it is explained in Chapter 9 of [5] and the reference therein. In this paper we study the cost of null controllability for transport-diffusion equations with Neumann, Robin and mixed boundary conditions.

Let $\Omega$ be a domain (a bounded connected open set of $\mathbb{R}^{d}$ ), $\Gamma \subset \partial \Omega$ be a relative open subset and $\Gamma^{*}:=\partial \Omega \backslash \Gamma$. We consider a family of functions $\left(a^{\varepsilon}(x)\right)_{\varepsilon \in\left(0, \varepsilon_{0}\right)} \subset L^{\infty}(\Gamma)$ for some $\varepsilon_{0}>0$. We study the control problem given by:

$$
\begin{cases}y_{t}-\varepsilon \Delta y+\partial_{x_{1}} y=1_{\omega} f, & \text { in } Q, \\ \partial_{n} y+a^{\varepsilon} y=0, & \text { on } \Sigma_{N}, \\ y=0, & \text { on } \Sigma_{D}, \\ y(0, \cdot)=y^{0}, & \text { on } \Omega,\end{cases}
$$

Keywords and phrases: Heat equation, singular limits, spectral decomposition, transport equation, uniform controllability.

${ }^{1}$ Departamento de Matemáticas, Universidad Autónoma de Madrid, 28049 Madrid, Spain.

2 Chair of Computational Mathematics, Fundación Deusto, Avenida de las Universidades 24, 48007 Bilbao, Basque Country, Spain.

* Corresponding author: jonasier.barcena@ehu.eus 
for $\varepsilon \in\left(0, \varepsilon_{0}\right)$, and in particular the cost of the control when $\varepsilon \rightarrow 0$. As usual, $n$ denotes the outward normal vector on $\partial \Omega, Q:=(0, T) \times \Omega, \omega \subset \Omega$ is an open subset, $Q_{\omega}:=(0, T) \times \omega$ and $\Sigma:=(0, T) \times \partial \Omega$. In addition, we define $\Sigma_{N}:=(0, T) \times \Gamma$ and $\Sigma_{D}:=(0, T) \times \Gamma^{*}$. The fact that (1.1) is well-posed is a classical result, which follows from Galerkin approximations, integrations by parts and Gronwall inequality (see [27] for a proof). The null controllability of (1.1) is already proved in [17] for $\Gamma=\partial \Omega$ and a function $a^{\varepsilon}(x) \in L^{\infty}(\partial \Omega)$ when $\varepsilon>0$ is fixed, though it can be adapted easily for the general case (see Rem. 3.6). However, those proofs do not provide accurate information of the cost of the control when $\varepsilon \rightarrow 0$. Understanding the bounds of the cost with respect to $\varepsilon$ is the main objective of this paper. In particular, we study the cost of the null controllability with the usual norms, that is,

$$
K(\Omega, \omega, T, \varepsilon):=\sup _{y^{0} \in L^{2}(\Omega) \backslash\{0\}} \inf _{f \in S\left(y^{0}\right)} \frac{\|f\|_{L^{2}\left(Q_{\omega}\right)}}{\left\|y^{0}\right\|_{L^{2}(\Omega)}},
$$

for:

$$
S\left(y^{0}\right):=\left\{f \in L^{2}\left(Q_{\omega}\right): \text { the solution of }(1.1) \text { satisfies } y(T, \cdot)=0\right\}
$$

The cost also depends on $a^{\varepsilon}$ and $\Gamma$, although we do not write it down explicitly.

When $T$ is small the cost of the null controllability of (1.1) explodes exponentially if $\varepsilon \rightarrow 0$, which can be proved, for instance, as in [24] and [2]. Thus, in this paper we focus on large values of $T$. First, we prove that if we almost have Dirichlet boundary conditions in the part of the boundary in which the flux of the transport enters, the cost of the controllability decays for a sufficiently large time $T$. Next, we show some examples of Neumann and mixed boundary conditions in which for any time $T>0$ the cost explodes exponentially as the diffusion coefficient $\varepsilon$ vanishes. Finally, we study the cost of the problem when the control is localized in the whole domain.

This paper follows a well-established research line which inquires about the cost of the null controllability of systems with a small diffusion and a transport term. The first of such control problems was the heat equation in dimension one with Dirichlet boundary conditions in [14]. Afterwards, the same problem but in any dimension and with a transport flow belonging to $W^{1, \infty}\left(\mathbb{R}^{+} \times \Omega\right)$ was studied in [24]. More recently, better approximations have been given for the optimal time in which the cost of the control decays: the lower bound was improved first in [34] through complex analysis and properties of the entire functions, and more recently in [28] through semi-classical and spectral analysis; and the upper bound was improved in $[20,32]$ (in the first one through complex analysis and, in the second one, by transforming the transport-diffusion equation into a heat equation with just a diffusive term). As for similar results, several results have been obtained for the KdV equation (see $[6,7,9,22,23]$ ), the Burgers equation (see [21]), the Stokes system (see [2]), an artificial advection-diffusion problem (see $[11,12]$ ), the heat equation on networks (see [3]), and a fourth-order parabolic equation (see $[8,26,35])$. Finally, the current study of $(1.1)$ is a contribution to the literature as it seeks to understand the evolution of the cost of the null controllability of the transport-diffusion equation with a large variety of boundary conditions when the diffusivity vanishes (and, in particular, the case $a^{\varepsilon}=0$ and $\Gamma=\emptyset$ was an open problem proposed in Remark 3 of [24]).

\section{QuANTIFicAtion OF THE MAin RESUlts}

In this section we first introduce the adjoint system, then symmetrize it, and finally present the controllability results. Most of these results are stated for $\varepsilon$ small enough, which means that there is some $\varepsilon_{0}^{\prime} \in\left(0, \varepsilon_{0}\right)$ such that these results are true for all $\varepsilon \in\left(0, \varepsilon_{0}^{\prime}\right)$. Indeed, as we are interested in obtaining estimates on the cost of the control when $\varepsilon$ vanishes, we can assume that $\varepsilon$ is small. 


\subsection{The observability problem and the symmetrized system}

As usual, we obtain the cost of the control by studying the observability of the adjoint system, which is given by:

$$
\begin{cases}-\varphi_{t}-\varepsilon \Delta \varphi-\partial_{x_{1}} \varphi=0, & \text { in } Q, \\ \varepsilon \partial_{n} \varphi+\left(\varepsilon a^{\varepsilon}+n_{1}\right) \varphi=0, & \text { on } \Sigma_{N}, \\ \varphi=0, & \text { on } \Sigma_{D}, \\ \varphi(T, \cdot)=\varphi^{T}, & \text { on } \Omega,\end{cases}
$$

for $n_{1}$ the first component of the vector $n$. Indeed, we have the classical equality given by the Hilbert Uniqueness Method (see, for instance, $[30,36])$ :

$$
K(\Omega, \omega, T, \varepsilon)=\sup _{\varphi^{T} \in L^{2}(\Omega) \backslash\{0\}} \frac{\|\varphi(0, \cdot)\|_{L^{2}(\Omega)}}{\|\varphi\|_{L^{2}\left(Q_{\omega}\right)}},
$$

where $\varphi$ denotes the solution of (2.1).

As a novelty with respect to the literature in systems with small diffusion and a transport term, we obtain some properties of (2.1) (and, in particular, the Carleman inequality) by studying the solutions of a system whose elliptic operator is self-adjoint. For that, we consider the map:

$$
w \mapsto w e^{(2 \varepsilon)^{-1} x_{1}} .
$$

Indeed, $\phi=\varphi e^{x_{1}(2 \varepsilon)^{-1}}$ is the solution of the following system:

$$
\begin{cases}-\phi_{t}-\varepsilon \Delta \phi+\frac{1}{4 \varepsilon} \phi=0, & \text { in } Q, \\ \varepsilon \partial_{n} \phi+\left(\varepsilon a^{\varepsilon}+\frac{n_{1}}{2}\right) \phi=0, & \text { on } \Sigma_{N}, \\ \phi=0, & \text { on } \Sigma_{D}, \\ \phi(T, \cdot)=\phi^{T}, & \text { on } \Omega .\end{cases}
$$

The motivation for (2.3) is to have an elliptic operator without first order term, as it is a necessary condition for an elliptic operator to be self-adjoint.

Remark 2.1. The map (2.3) is a homeomorphism from the solutions of $(2.1)$ with initial value in $L^{2}(\Omega)$ to the solutions of (2.4) with initial value in $L^{2}(\Omega)$. This homeomorphism is useful to translate information about the solutions of (2.4) to information about the solutions of (2.1), for instance about their regularity.

With the purpose of understanding the solutions of (2.1) we also study the spectral problem:

$$
\begin{cases}-\varepsilon \Delta u-\partial_{x_{1}} u=\lambda u, & \text { in } \Omega, \\ \varepsilon \partial_{n} u+\left(\varepsilon a^{\varepsilon}+n_{1}\right) u=0, & \text { on } \Gamma, \\ u=0, & \text { on } \Gamma^{*} .\end{cases}
$$

In that sense, with the purpose of understanding the solutions of (2.5) we study the spectral problem:

$$
\begin{cases}-\Delta v=\tilde{\lambda} v, & \text { in } \Omega, \\ \varepsilon \partial_{n} v+\left(\varepsilon a^{\varepsilon}+\frac{n_{1}}{2}\right) v=0, & \text { on } \Gamma, \\ v=0, & \text { on } \Gamma^{*},\end{cases}
$$


and we define:

$$
\mathcal{D}_{\varepsilon}:=\left\{w \in H^{1}(\Omega):-\Delta w \in L^{2}(\Omega), \varepsilon \partial_{n} w+\left(\varepsilon a^{\varepsilon}+\frac{n_{1}}{2}\right) w=0 \text { on } \Gamma, w=0 \text { on } \Gamma^{*}\right\} .
$$

We recall that $-\Delta w \in L^{2}(\Omega)$ and $w \in H^{1}(\Omega)$ imply that $\partial_{n} w$ is well-defined as an element of $H^{-1 / 2}(\partial \Omega)$. We consider the first equation of (2.6) instead of $-\varepsilon \Delta v+(4 \varepsilon)^{-1} v=\lambda v$ in order to isolate the "part" of the eigenvalue that is caused by the diffusion, which is the meaning of $\tilde{\lambda}$. This is useful for instance in Lemmas 3.3 and 5.1 below.

Remark 2.2. We can relate the solutions of (2.5) and (2.6) with the map (2.3). Indeed, we have that $(v, \tilde{\lambda})$ is a solution of (2.6) if and only if

$$
\left(v e^{-(2 \varepsilon)^{-1} x_{1}}, \varepsilon \tilde{\lambda}+\frac{1}{4 \varepsilon}\right)
$$

is a solution of (2.5). Similarly, $(u, \lambda)$ is a solution of (2.5) if and only if

$$
\left(u e^{(2 \varepsilon)^{-1} x_{1}}, \frac{\lambda}{\varepsilon}-\frac{1}{4 \varepsilon^{2}}\right)
$$

is a solution of (2.6).

Remark 2.3. It can be checked directly that the Laplacian is symmetric in $\mathcal{D}_{\varepsilon}$ for any Lipschitz domain $\Omega$. In addition, for a constant $C$ sufficiently large depending on $\varepsilon$ we have that $(-\Delta+C)^{-1}$ is compact. Thereby, $L^{2}(\Omega)$ has a spectral decomposition for each $\varepsilon$ by the solutions of (2.6) (see, for instance, [16], Appendix D). This implies, among other things, that (2.4) is a well-posed system, and consequently so is (2.1).

Remark 2.4. We can also study the problem with null initial force associated to (1.1). Indeed, the map $w \mapsto e^{-(2 \varepsilon)^{-1} x_{1}} w$ transforms (1.1) into (2.4) but forward in time. Thus, the dynamics and in particular the decay properties of the direct and adjoint systems are similar.

Definition 2.5. We denote by $\left(\lambda_{m}^{\varepsilon}\right)_{m \in \mathbb{N}}$ the eigenvalues of $(2.5)$ and by $\left(\tilde{\lambda}_{m}^{\varepsilon}\right)_{m \in \mathbb{N}}$ the eigenvalues of $(2.6)$ (counting multiplicities in both cases). In addition, for each eigenvalue of (2.6) an eigenfunction $v_{m}^{\varepsilon}$ which satisfies $\left\|v_{m}^{\varepsilon}\right\|_{L^{2}(\Omega)}=1$ is fixed. Finally, we denote $u_{m}^{\varepsilon}:=v_{m}^{\varepsilon} e^{-(2 \varepsilon)^{-1} x_{1}}$.

Remark 2.6. Since $\overline{\operatorname{span}\left\{v_{m}^{\varepsilon}\right\}}=L^{2}(\Omega)$ (see Rem. 2.3) we have that $\overline{\operatorname{span}\left\{u_{m}^{\varepsilon}\right\}}=L^{2}(\Omega)$.

To determine if the solutions of (2.1) decay we focus on $\tilde{\lambda}_{0}^{\varepsilon}$. We recall that by Rayleigh principle we have the equality:

$$
\tilde{\lambda}_{0}^{\varepsilon}=\min \left\{\int_{\Omega}|\nabla v|^{2} \mathrm{~d} x+\int_{\Gamma}\left(a^{\varepsilon}+\frac{n_{1}}{2 \varepsilon}\right)|v|^{2} \mathrm{~d} x: v \in H^{1}(\Omega),\|v\|_{L^{2}(\Omega)}=1, v=0 \text { on } \Gamma^{*}\right\} .
$$

From (2.8) we can distinguish two main cases depending on whether the following inequality is satisfied for $\varepsilon$ small enough:

$$
\left(a^{\varepsilon}+(2 \varepsilon)^{-1} n_{1}\right) 1_{\Gamma} \geq 0 .
$$

Indeed, if (2.9) is satisfied for $\varepsilon$ small enough the cost of the controllability decays when $\varepsilon \rightarrow 0$, whereas if (2.9) is not true the cost may explode for a sufficiently large time. The fact that the behaviour and controllability properties of the solutions of (1.1) varies so much when changing $a^{\varepsilon}$ implies that we cannot expect a general result that works for any arbitrary family $\left(a^{\varepsilon}\right)_{\varepsilon \in\left(0, \varepsilon_{0}\right)}$ unless it has some structure with respect to $\varepsilon$. 
The idea of using a spectral decomposition is not new in Control Theory (see, for instance, [13]). Indeed, for the heat equation alone, there are many papers which deal with eigenfunctions of the elliptic operator for proving the existence of some control (see, for example, [29] for Dirichlet boundary conditions and [4] for a coupled heat equation system with Robin boundary conditions) and for estimating the cost of the control (see, for instance, [15]). Similarly, for the Stokes system this technique was used to prove the existence of a control (see, for example, [10]) and for providing a negative answer to the existence of a control (see, for instance, [31]). As for a system with a small diffusion and a transport term, a spectral decomposition indirectly appears in $[14,34]$, when the authors obtain lower bounds for the optimal time $T_{0}$ in which the cost of the control decays exponentially with $\varepsilon$. More recently, it has been used in [28] to prove estimates about the optimal time in which the heat equation with a transport term decays when $\varepsilon \rightarrow 0$. In addition, a spectral decomposition has been used in [2] to obtain the dissipation estimate in a transport-diffusion Stokes system. Indeed, in this paper we follow the philosophy of [2] of using as much information as possible about the spectral decomposition, with the contribution that now while proving the Carleman estimate we work directly in the symmetrized system, that is, in (2.4).

The idea of transforming one system into another by using a family of isomorphism similar to (2.3) is not new. In particular, these or similar transformations have been used for instance in [20], [32], [33] and [34] to obtain bounds of the optimal time in which the cost of the control decays when $\varepsilon \rightarrow 0$ in the transport-diffusion equation with Dirichlet boundary conditions. However, to prove these bounds the authors either work in a one-dimensional domain or require the geometric control condition for the wave equation.

\subsection{The regime in which the cost of the control decays exponentially}

In this section we study the case in which (2.9) is satisfied for $\varepsilon$ small enough. In particular, we prove that:

Theorem 2.7. Let $\Omega$ be a $C^{2}$ domain, $\omega \subset \Omega$ be a subdomain and assume that $\left(\Gamma, a^{\varepsilon}\right)$ satisfies that $a^{\varepsilon} \in L^{\infty}(\Gamma)$ and (2.9) for $\varepsilon$ small enough. Then, there are $T_{0}, c, C>0$ depending only on $\omega$ and $\Omega$ such that for $\varepsilon$ small enough and all $T \geq T_{0}$ we have that:

$$
K(\Omega, \omega, T, \varepsilon) \leq C e^{-c \varepsilon^{-1}} .
$$

The proof of Theorem 2.7 is given in Section 3. In particular, we first prove a dissipation estimate for the solutions of (2.1) under the assumptions of Theorem 2.7, and then prove a Carleman inequality.

There are several important examples that satisfy the hypotheses assumed in Theorem 2.7:

- Dirichlet boundary conditions $(\Gamma=\emptyset)$. This controllability result is already proved in [14, 24]. However, our proof is different since we prove the decay with the spectral decomposition (instead of with a comparison theorem or an Agmon inequality) and we prove the Carleman inequality for (2.4) rather than for (2.1).

- Segments in which we have Dirichlet boundary conditions on the left end and Neumann boundary conditions on the right end.

- Any system in which $a^{\varepsilon} \geq 0$ and $n_{1} 1_{\Gamma} \geq 0$, that is, in which we have Dirichlet boundary conditions on the part of the boundary in which the flux of the transport enters and either Dirichlet or Robin with a positive coefficient on the other part of the boundary.

- Any system in which we almost have Dirichlet boundary conditions on the part of the boundary in which the flux of the transport enters and either Dirichlet or Robin with a coefficient whose negative part is not too large on the other part of the boundary.

\subsection{The regime in which the cost of the control explodes exponentially}

In this section we study the case in which (2.9) is not satisfied, and in particular some situations in which:

$$
\operatorname{measure}_{\mid \partial \Omega}\left(\left(a^{\varepsilon}+\frac{n_{1}}{2 \varepsilon}\right) 1_{\Gamma}<0\right)>0
$$


for $\varepsilon$ small enough. Of course, we refer to the Hausdorff measure. Under the hypothesis (2.11) we might have eigenvalues of (2.5) close to 0, null, or negative, so we may have no dissipation making the cost of the control explode. The results of this section are proved in Section 4.

First, we consider (1.1) with pure Neumann boundary conditions, that is, $a^{\varepsilon}=0$ and $\Gamma=\partial \Omega$. We remark that under those boundary conditions (1.1) is given by:

$$
\begin{cases}y_{t}-\varepsilon \Delta y+\partial_{x_{1}} y=1_{\omega} f, & \text { in } Q, \\ \partial_{n} y=0, & \text { on } \Sigma, \\ y(0, \cdot)=y^{0}, & \text { on } \Omega .\end{cases}
$$

The study of the cost of the null controllability of (2.12) is an open problem proposed in Remark 3 of [24]. Let us denote:

$$
p_{l}:=\inf \pi_{1}(\Omega), \quad p_{r}:=\sup \pi_{1}(\Omega)
$$

for $\pi_{1}(x)=x_{1}$, i.e. the projection on the first coordinate. We prove in this paper that the cost of the control of (2.12) explodes exponentially:

Theorem 2.8. Let $h>0, \Omega$ be a domain, and $\omega \subset \Omega$ be an open subset such that:

$$
\pi_{1}(\omega) \subset\left(p_{l}+h, p_{r}\right)
$$

Then, for all $T>0$ there is $c>0$ depending on $h$ and $T$ such that for all $\varepsilon>0$ :

$$
K(\Omega, \omega, T, \varepsilon) \geq c e^{c \varepsilon^{-1}},
$$

for $K$ the cost of the null controllability of (2.12).

Theorem 2.8 is similar to the result presented in [2] for the Stokes equation in dimension 3. The reason why Theorem 2.8 is true is that the cost of controlling $\pi_{1}^{-1}\left(p_{l}, \inf \pi_{1}(\omega)\right)$ explodes exponentially. Indeed, because of the Neumann boundary conditions the solution does not decay by just switching off the control when the viscosity vanishes. In addition, due to the vanishing viscosity and the direction of the transport flow, the effect of the control on $\pi_{1}^{-1}\left(p_{l}\right.$, inf $\left.\pi_{1}(\omega)\right)$ decays exponentially. In particular, the hypothesis (2.14) is necessary as the conclusion of Theorem 2.8 is false for $\omega=\Omega$ (see Prop. 2.16). The key point of the proof is to study the spatial repartition of the steady solution of the adjoint system given by the first eigenfunction.

Next, we consider a segment with Neumann boundary conditions on the left edge of the segment and Dirichlet boundary conditions on its right edge, that is, $\Omega:=(-L, 0), \Gamma:=\{-L\}$ and $a^{\varepsilon}=0$. In this situation the control problem (1.1) is given by:

$$
\begin{cases}y_{t}-\varepsilon \partial_{x x} y+\partial_{x} y=f 1_{\omega}, & \text { in } Q \\ \partial_{x} y(\cdot,-L)=0, & \text { on }(0, T), \\ y(\cdot, 0)=0, & \text { on }(0, T), \\ y(0, \cdot)=y^{0}, & \text { on }(-L, 0)\end{cases}
$$

The intuition may suggest that as $\varepsilon$ vanishes the cost of the control decreases, as in the Dirichlet case. However, it is just the opposite: the fact that there is a flux coming from the left dominates and the cost of the control actually increases when the diffusivity vanishes. In particular, we prove that: 
Theorem 2.9. Let $L, h>0, \Omega=(-L, 0)$ and $\omega \subset(-L+h, 0)$ be an open subset. Then, for all $T>0$ there is $c>0$ depending on $T, L$ and $h$ such that for $\varepsilon$ small enough we have the estimate:

$$
K(\Omega, \omega, T, \varepsilon) \geq c e^{c \varepsilon^{-1}},
$$

for $K$ the cost of the null controllability of (2.16).

As in Theorem 2.8, the reason why Theorem 2.9 is true is that the cost of controlling $(-L$, inf $\omega)$ explodes exponentially and the key point of the proof is to look at the first eigenfunction. In addition, the hypothesis of $\omega \subset(-L+h, 0)$ is necessary as the conclusion of Theorem 2.9 is false for $\omega=\Omega$ (see Prop. 2.16).

In both Theorems 2.8 and 2.9 the cost of the control problem explodes because of the interaction of the transport with the boundary conditions. Indeed, as a consequence of the following proposition the solutions of (2.1) when $a^{\varepsilon} \geq 0$ (which is satisfied in Thms. 2.8 and 2.9) do not explode when $T \rightarrow \infty$ :

Proposition 2.10. Let $\Omega$ be a Lipschitz domain and $a^{\varepsilon} \geq 0$. Then, we have that:

$$
\lambda_{0}^{\varepsilon} \geq 0
$$

In addition, we have $\lambda^{\varepsilon}=0$ if and only if $a^{\varepsilon}=0$ and $\Gamma=\partial \Omega$, and in that case the associated eigenfunction is $u^{\varepsilon}=\frac{e^{-\varepsilon^{-1} x_{1}}}{\left\|e^{-\varepsilon^{-1} x_{1} / 2}\right\|_{L^{2}(\Omega)}}$.

Remark 2.11. The equality case in Proposition 2.10 corresponds to the control problem (2.12).

\subsection{Controllability results for controls acting on the boundary}

In this section we state the results when the control is on the boundary:

Theorem 2.12. Let $\Omega$ be a $C^{2}$ domain, $\gamma \subset \Gamma^{*}$ be relatively open and assume that $\left(\Gamma, a^{\varepsilon}\right)$ satisfies (2.9) for $\varepsilon$ small enough. Then, there are $T_{0}, c, C>0$ depending only on $\gamma$ and $\Omega$ such that for $\varepsilon$ small enough and $T \geq T_{0}$ we have that:

$$
K(\Omega, \gamma, T, \varepsilon) \leq C e^{-c \varepsilon^{-1}}
$$

for $K$ the cost of the null controllability of:

$$
\begin{cases}y_{t}-\varepsilon \Delta y+\partial_{x_{1}} y=0, & \text { in } Q, \\ \partial_{n} y+a^{\varepsilon} y=0, & \text { on } \Sigma_{N}, \\ y=f 1_{\gamma}, & \text { on } \Sigma_{D}, \\ y(0, \cdot)=y^{0}, & \text { on } \Omega .\end{cases}
$$

Theorem 2.13. Let $h>0, \Omega$ be a domain, and $\gamma \subset \partial \Omega$ be a relatively open subset such that:

$$
\pi_{1}(\gamma) \subset\left(p_{l}+h, p_{r}\right]
$$

Then, for all $T>0$ there is $c>0$ depending on $h$ and $T$ such that for all $\varepsilon>0$ :

$$
K(\Omega, \gamma, T, \varepsilon) \geq c e^{c \varepsilon^{-1}}
$$


for $K$ the cost of the null controllability of:

$$
\begin{cases}y_{t}-\varepsilon \Delta y+\partial_{x_{1}} y=0, & \text { in } Q, \\ \partial_{n} y=f 1_{\gamma}, & \text { on } \Sigma, \\ y(0, \cdot)=y^{0}, & \text { on } \Omega .\end{cases}
$$

Theorem 2.14. Let $L>0, \Omega=(-L, 0)$. Then, for all $T>0$ there is $c>0$ depending on $T$ and $L$ such that for $\varepsilon$ small enough we have the estimate:

$$
K(\Omega, T, \varepsilon) \geq c e^{c \varepsilon^{-1}}
$$

for $K$ the cost of the the null controllability of:

$$
\begin{cases}y_{t}-\varepsilon \partial_{x x} y+\partial_{x} y=0, & \text { in } Q, \\ \partial_{x} y(\cdot,-L)=0, & \text { on }(0, T), \\ y(\cdot, 0)=f, & \text { on }(0, T), \\ y(0, \cdot)=y^{0}, & \text { on }(-L, 0) .\end{cases}
$$

The proof of Theorem 2.12 is very similar to that of Theorem 2.7. In addition, the proofs of Theorems 2.13 and 2.14 are analogous to those of Theorems 2.8 and 2.9. Thus, these proofs are omitted.

\subsection{Cost of the control when $\omega=\Omega$}

In this section we prove a lower bound for the cost of the null controllability when $\omega=\Omega$, that is, in which the control domain is the whole domain. It is well-known that if we have a control in the whole domain the system will be controllable, even with a regular control (for that, it suffices to let the system evolve freely in $(0, t)$ and then considering the segment in $H^{2}(\Omega)$ which joins the state at time $t$ and 0$)$. However, what is not obvious and makes the following results interesting is to determine how the cost of the control depends on $\varepsilon$ and $T$. In addition, with these results we show that $(2.14)$ and $\omega \subset(-L+h, 0)$ are necessary hypotheses for Theorems 2.8 and 2.9. The results of this section are proved in Section 5.

We first prove a lower bound for the cost of the control:

Proposition 2.15. Let $\Omega$ be a Lipschitz domain, $T>0$ and $\left(\Gamma, a^{\varepsilon}\right)$ satisfying:

$$
\lim _{\varepsilon \rightarrow 0} \lambda_{0}^{\varepsilon}=0
$$

Then, we have the bound:

$$
\liminf _{\varepsilon \rightarrow 0} K(\Omega, \Omega, T, \varepsilon) \geq \frac{1}{\sqrt{T}}
$$

The condition stated in Proposition 2.15 includes the control problems (2.12) and (2.16), as it is implied by Proposition 2.10 and (4.16) in Section 4.3 respectively. Proposition 2.15 is proved just by an easy direct computation on the first frequency.

Next, we prove an upper bound for the cost of the control. In particular if $a^{\varepsilon} \geq 0$, which includes Neumann boundary conditions, we prove that: 
Proposition 2.16. Let $\Omega$ be a $C^{2}$ domain and $a^{\varepsilon} \geq 0$. Then, there is $C>0$ dependent on $\Omega$ such that for all $\varepsilon>0$ and $T>0$ :

$$
K(\Omega, \Omega, T, \varepsilon) \leq C\left(\frac{1}{\sqrt{T}}+\frac{1}{\sqrt{\varepsilon}}\right)
$$

for $K$ the cost of the null controllability of (1.1).

The proof of Proposition 2.16 relies on trace and energy estimates. In addition, for Neumann boundary conditions we prove in segments a more precise result:

Proposition 2.17. Let $L>0$ and $\Omega=(-L, 0)$. We have for all $T \geq 4 L$ :

$$
\limsup _{\varepsilon \rightarrow 0} K(\Omega, \Omega, T, \varepsilon) \leq \frac{4}{\sqrt{T}}
$$

for $K$ the cost of the null controllability of (1.1) with $a^{\varepsilon}=0$.

Proposition 2.17 is proved by explicit computations of the solutions and a splitting between the first frequency, which remains constant, and the others, which dissipate exponentially.

Remark 2.18. The difficulty when proving Propositions 2.16 and 2.17 is that the cost of the control with norms $L^{2}$ must be bounded when $\varepsilon \rightarrow 0$. If we just look for controls in $L^{2}\left(0, T ; H^{-2}(\Omega)\right)$, then it suffices to consider the affine trajectory joining $y^{0}$ and 0 . Similarly, we can obtain a control $f \in L^{2}(Q)$ by setting $f=0$ on $(0, t)$ for $t>0$ a small value, and then consider the affine function which joins $y(t, 0)$ and 0 . However, this is not an optimal way to obtain a control.

The rest of the paper is organized as follows: in Section 3 we prove Theorem 2.7, in Section 4 we prove Proposition 2.10 and Theorems 2.8 and 2.9, in Section 5 we prove Propositions 2.15, 2.16 and 2.17 and, finally, in Section 6 we present some open problems.

Notation. In this paper we denote $\operatorname{diam}_{x_{1}}(\Omega):=\sup \pi_{1}(\Omega)-\inf \pi_{1}(\Omega)$. The interest of this notation is that $x_{1}$ is the direction of the flow of the transport. Also, the operator $|\cdot|$ denotes the volume of a domain. In addition $\mathcal{D}(\Omega):=\left\{u \in C^{\infty}(\bar{\Omega}): \operatorname{supp}(u) \subset \Omega\right\}$. Finally, $C>0$ denotes a generic positive constant that may depend on some parameters as specified in the statement of each result and that changes from line to line.

\section{Some CONTROLlability PROBlems in Which THE COST OF THE CONTROL DECAYS EXPONENTIALLY}

In this section we prove Theorem 2.7. In particular, in Section 3.1 we prove the decay of the solutions of (2.1), in Section 3.2 we prove a Carleman inequality for the solutions of (2.4), and in Section 3.3 we combine both results and conclude the proof of Theorem 2.7 .

\subsection{Dissipation of the solutions of (2.1) for a sufficiently large time}

We denote by $\left(\tilde{\lambda}_{m}^{0}\right)_{m \in \mathbb{N}}$ the eigenvalues of the the Neumann Laplacian, which we recall are nonnegative and satisfy Weyl's law (see, for instance, [25]):

Lemma 3.1 (Weyl's law). Let $\Omega$ be a Lipschitz domain and let $N(\gamma)$ be the number of eigenvalues (counting repetitions) of the Neumann Laplacian that belong to $(-\infty, \gamma]$. We have:

$$
\lim _{\gamma \rightarrow \infty} \frac{N(\gamma)}{\gamma^{d / 2}}=\frac{|B(0,1)||\Omega|}{(2 \pi)^{d}}
$$


for $d$ the dimension of $\Omega$. In particular, there is $C>0$ depending on $\Omega$ such that for all $\gamma \in \mathbb{R}$ :

$$
N(\gamma) \leq C\left(1+|\gamma|^{d / 2}\right)
$$

To continue with, we highlight the following result:

Lemma 3.2. Let $\Omega$ be a Lipschitz domain, $\varepsilon>0$ and assume that $\left(\Gamma, a^{\varepsilon}\right)$ satisfies (2.9). Then, for all $m \in \mathbb{N}$ we have the following estimate:

$$
\tilde{\lambda}_{m}^{\varepsilon} \geq \tilde{\lambda}_{m}^{0}
$$

Lemma 3.2 can be obtained by comparing $\tilde{\lambda}_{m}^{\varepsilon}$ (see Def. 2.5) with $\tilde{\lambda}_{m}^{0}$ by using the min-max variational principle

Let us now study the solutions of (2.1):

Lemma 3.3. Let $\Omega$ be a Lipschitz domain, $\varepsilon>0$ and assume that $\left(\Gamma, a^{\varepsilon}\right)$ satisfies (2.9). Then, for all $\varphi^{T} \in$ $L^{2}(\Omega)$ the solution of $(2.1)$ satisfies in $C^{0}\left([0, T] ; L^{2}(\Omega)\right)$ :

$$
\varphi(t, x)=\sum_{m \in \mathbb{N}}\left(\int_{\Omega} \varphi^{T}(z) e^{(2 \varepsilon)^{-1} z_{1}} v_{m}^{\varepsilon}(z) \mathrm{d} z\right) v_{m}^{\varepsilon}(x) e^{-(2 \varepsilon)^{-1} x_{1}} \exp \left[-\left(\varepsilon \tilde{\lambda}_{m}^{\varepsilon}+\frac{1}{4 \varepsilon}\right)(T-t)\right] .
$$

In particular, the series in the right-hand side of (3.2) is absolutely convergent in $L^{2}(\Omega)$ for all $t<T$. Moreover, for all $T_{0}>0$ and $\delta>0$ there is $C>0$ depending on $\Omega, \delta$ and $T_{0}$ such that for all $T \geq T_{0}, \varphi^{T} \in L^{2}(\Omega)$ and $t \in\left[0, T-T_{0}\right]$ we have the estimate:

$$
\|\varphi(t, \cdot)\|_{L^{2}(\Omega)} \leq C\left\|\varphi^{T}\right\|_{L^{2}(\Omega)} \exp \left(\frac{2 \operatorname{diam}_{x_{1}}(\Omega)+\delta-(T-t)}{4 \varepsilon}\right) .
$$

Proof. We can easily prove (3.2) for $t=T$ using that $v_{m}^{\varepsilon}$ is an orthonormal basis in $L^{2}(\Omega)$. Indeed, if $\varphi^{T} \in L^{2}(\Omega)$ we have that:

$$
\varphi^{T}(x)=\left(\varphi^{T}(x) e^{(2 \varepsilon)^{-1} x_{1}}\right) e^{-(2 \varepsilon)^{-1} x_{1}}=\sum_{m \in \mathbb{N}}\left(\int_{\Omega} \varphi^{T}(z) e^{(2 \varepsilon)^{-1} z_{1}} v_{m}^{\varepsilon}(z) \mathrm{d} z\right) v_{m}^{\varepsilon}(x) e^{-(2 \varepsilon)^{-1} x_{1}} .
$$

We prove (3.2) and (3.3) simultaneously for all $t \in[0, T)$. We obtain from Remark 2.2 that (3.2) is true in $\operatorname{span}\left\{u_{m}^{\varepsilon}\right\}$. Since the left-hand side of $(3.2)$ belongs to $C^{0}\left([0, T] ; L^{2}(\Omega)\right)$ (see [17] and [27, Chapter III]) and since $\operatorname{span}\left\{u_{m}^{\varepsilon}\right\}_{m \in \mathbb{N}}$ is dense in $L^{2}(\Omega)$ (see Rem. 2.6), it is enough to prove that the right-hand side is an endomorphism on $L^{2}(\Omega)$ viewed as a map of pre-image $\varphi^{T}$ and time $t \in[0, T)$ fixed. For that purpose, using the triangle inequality and that $\left\|v_{m}^{\varepsilon}\right\|_{L^{2}(\Omega)}=1$, we obtain the following:

$$
\begin{array}{r}
\left\|\sum_{m \in \mathbb{N}}\left(\int_{\Omega} \varphi^{T}(z) e^{(2 \varepsilon)^{-1} z_{1}} v_{m}^{\varepsilon}(z) \mathrm{d} z\right) v_{m}^{\varepsilon}(x) e^{-(2 \varepsilon)^{-1} x_{1}} \exp \left[-\left(\varepsilon \tilde{\lambda}_{m}^{\varepsilon}+\frac{1}{4 \varepsilon}\right)(T-t)\right]\right\|_{L^{2}(\Omega, \mathrm{d} x)} \\
\leq\left\|\varphi^{T}\right\|_{L^{2}(\Omega)} \exp \left(\frac{2 \operatorname{diam}_{x_{1}}(\Omega)-(T-t)}{4 \varepsilon}\right) \sum_{m \in \mathbb{N}} e^{-\varepsilon \tilde{\lambda}_{m}^{\varepsilon}(T-t)}
\end{array}
$$

Thus, the only thing left is to bound $\sum_{m \in \mathbb{N}} e^{-\varepsilon \tilde{\lambda}_{m}^{\varepsilon}(T-t)}$. We obtain from Lemmas 3.2 and 3.1 and from the non-negativity of the eigenvalues of the Neumann Laplacian the estimate: 


$$
\begin{aligned}
\sum_{m \in \mathbb{N}} e^{-\varepsilon \tilde{\lambda}_{m}^{\varepsilon}(T-t)} & \leq \sum_{m \in \mathbb{N}} e^{-\varepsilon \tilde{\lambda}_{m}^{0}(T-t)} \leq \sum_{m \geq 1} N(m) e^{-\varepsilon(m-1)(T-t)} \\
\leq C & \left(1+\sum_{m \geq 2}\left(1+m^{d / 2}\right) e^{-\varepsilon(m-1)(T-t)}\right) \leq C\left(1+\int_{0}^{+\infty}\left(1+(z+2)^{d / 2}\right) e^{-\varepsilon z(T-t)} \mathrm{d} z\right) \\
& \leq C\left(1+\int_{0}^{+\infty}\left(1+z^{d / 2}\right) e^{-\varepsilon z(T-t)} \mathrm{d} z\right) \leq C\left(1+\frac{1}{\varepsilon(T-t)}+\frac{1}{\varepsilon^{(d+2) / 2}(T-t)^{(d+2) / 2}}\right) .
\end{aligned}
$$

So, from (3.5), $T-t \geq T_{0}$, and $z^{-k / 2} e^{-z^{-1}} \in L^{\infty}\left(\mathbb{R}^{+}\right)$for all $k \in \mathbb{R}^{+}$, we obtain that:

$$
\sum_{m \in \mathbb{N}} e^{-\varepsilon \tilde{\lambda}_{m}^{\varepsilon}(T-t)} \leq C e^{\delta \varepsilon^{-1}}
$$

Consequently, we end the proof by combining (3.4) and (3.6).

Remark 3.4. Lemma 3.3 can be generalized to the case in which $\tilde{\lambda}_{0}^{\varepsilon} \geq 0$ and there are some polynomial bound for $\left|\left\{i: \tilde{\lambda}_{i}^{\varepsilon} \leq m\right\}\right|$ that depends on $m$ and uniform for all $\varepsilon \in\left(0, \varepsilon_{0}\right)$.

\subsection{A Carleman inequality for the solutions of $(2.4)$}

We prove in this section a Carleman inequality for (2.4) when $\Omega$ is $C^{2}$. First of all, we consider $\eta$ a $C^{2}(\bar{\Omega})$ function satisfying:

$$
\eta=0 \text { on } \partial \Omega, \quad \eta \geq 0 \text { in } \Omega, \quad\|\eta\|_{\infty}=1, \quad \inf _{\Omega \backslash \omega_{0}}|\nabla \eta|=\delta>0
$$

for $\omega_{0} \subset \subset \omega$ an open non-empty set. The existence of such a function if $\Omega$ is a $C^{2}$ domain is proved, for instance, in [19]. With that auxiliary function in mind, we define for some $\tilde{T}>0$ the following weights in $\tilde{Q}:=(0, \tilde{T}) \times \Omega$ :

$$
\alpha_{ \pm}(t, x):=\frac{e^{8 \tau}-e^{\tau(6 \pm \eta(x))}}{t(\tilde{T}-t)}, \quad \xi_{ \pm}(t, x):=\frac{e^{\tau(6 \pm \eta(x))}}{t(\tilde{T}-t)},
$$

for $\tau>0$ a large parameter to be fixed later on. Weights of this kind first appeared in [19], but this version is borrowed from [18] with the adaptations for equations in which we do not have Dirichlet boundary conditions proposed in [17]. We remark that we have the usual bounds:

$$
\left|\partial_{x_{i}} \alpha_{ \pm}\right|=\left|\partial_{x_{i}} \xi_{ \pm}\right| \leq C \tau \xi_{ \pm}, \quad\left|\partial_{t} \alpha_{ \pm}\right|+\left|\partial_{t} \xi_{ \pm}\right| \leq C \tilde{T} \xi_{ \pm}^{2}, \quad\left|\partial_{t t} \alpha_{ \pm}\right| \leq C \xi_{ \pm}^{2}\left(1+\tilde{T}^{2} \xi_{ \pm}\right) .
$$

We prove in this section the following result:

Proposition 3.5. Let $\Omega$ be a $C^{2}$ domain, $\omega \subset \Omega$ be a subdomain, $\tilde{T}>0, \varepsilon>0$ and assume that $\left(\Gamma, a^{\varepsilon}\right)$ satisfies (2.9). Then, there is $C>0$ depending on $\omega$ and $\Omega$ such that if $\tau \geq C, s \geq C\left(\tilde{T}+\tilde{T}^{2}\right) \varepsilon^{-1}$ and $\phi^{\tilde{T}} \in L^{2}(\Omega)$ we have that:

$$
\begin{aligned}
s^{3} \tau^{4} \iint_{\tilde{Q}} e^{-2 s \alpha_{+}} \xi_{+}^{3}|\phi|^{2}+s \tau^{2} \iint_{\tilde{Q}} e^{-2 s \alpha_{+}} \xi_{+}|\nabla \phi|^{2}+s^{2} \tau^{2} \iint_{\tilde{\Sigma}_{N}} e^{-2 s \alpha_{+}} \xi_{+}^{2} & \left(a^{\varepsilon}+\frac{n_{1}}{2 \varepsilon}\right)|\phi|^{2} \\
& \leq C s^{3} \tau^{4} \iint_{\tilde{Q}_{\omega}} e^{-2 s \alpha_{+}} \xi_{+}^{3}|\phi|^{2},
\end{aligned}
$$

for $\phi$ the solution of (2.4) with $\tilde{T}$ instead of $T, \tilde{\Sigma}_{N}:=(0, \tilde{T}) \times \Gamma$ and $\tilde{Q}_{\omega}:=(0, \tilde{T}) \times \omega$. 
In the statement of Proposition 3.5 and in its proof we omit the infinitesimals $d t$ and $\mathrm{d} x$ as they can be deduced by looking at the integration domain. In the proof of Proposition 3.5 we denote by $O(\mathcal{G}(\varepsilon, s, \tau, \psi))$ a generic function for which there is $C>0$ depending only on $\Omega, \omega$ and $\omega_{0}$ and which satisfies:

$$
|O(\mathcal{G}(\varepsilon, s, \tau, \psi))| \leq C \mathcal{G}(\varepsilon, s, \tau, \psi),
$$

if $\varepsilon$ is small enough, $\tau \geq C, s \geq C\left(\tilde{T}+\tilde{T}^{2}\right) \varepsilon^{-1}$ and if $\psi$ is a regular function. In that definition $\mathcal{G}$ is a nonnegative operator.

For proving Proposition 3.5 we adapt the proofs of [24] and [17]. In particular, many of the estimates here are inspired by Proposition 1 of [24], though we use the technique proposed in [17] to treat the boundary terms. However there are some differences. The main difference is on how we deal with the transport term: indeed, in [24] the authors prove the Carleman for the solutions of the adjoint system (i.e. (2.1)) instead of the symmetrized system (i.e. (2.4)). This difference is important to obtain $s^{2} \tau^{2} \iint_{\tilde{\Sigma}_{N}} e^{-2 s \alpha_{+}} \xi_{+}^{2}\left(a^{\varepsilon}+\frac{n_{1}}{2 \varepsilon}\right)|\phi|^{2}$ in the left-hand side of (3.10) and not a term like $\varepsilon \iint_{\Sigma} \partial_{x_{1}} \psi^{ \pm} \partial_{n} \psi^{ \pm}-\frac{\varepsilon}{2} \iint_{\Sigma} n_{1}\left|\nabla \psi^{ \pm}\right|^{2}$, which is not easily treatable. In addition, unlike in [24] and [17] the solutions of (2.4) may not belong to $L^{2}\left(0, T ; H^{2}(\Omega)\right)$ even if $\phi^{\tilde{T}} \in \mathcal{D}(\Omega)$. We first estimate a scalar product for any $\phi \in C^{\infty}(\bar{Q})$, which allows to do some integrations by parts involving the Laplacian, like in (3.19) below. Then, by using that $C^{\infty}(\bar{Q})$ is dense in $H^{1}\left(0, T ; \mathcal{D}_{\varepsilon}\right)$ (see $(2.7)$ ), we prove an estimate for the solutions of (2.4) with $\phi^{\tilde{T}} \in \mathcal{D}(\Omega)$. Next, we use the boundary conditions of the solutions of (2.4) to deal with the trace term. Finally we use the density of $\mathcal{D}(\Omega)$ in $L^{2}(\Omega)$ to prove (3.10) for all solutions of (2.4) with $\phi^{\tilde{T}} \in L^{2}(\Omega)$.

Proof. As in [17], we consider the following change of variables:

$$
\psi^{ \pm}:=e^{-s \alpha_{ \pm}} \phi
$$

Since $\eta=0$ on $\partial \Omega$ we have the following equations on $\tilde{\Sigma}:=(0, \tilde{T}) \times \partial \Omega$ :

$$
\xi_{+}=\xi_{-}, \quad \alpha_{+}=\alpha_{-}, \quad \psi^{+}=\psi^{-}, \quad \psi_{t}^{+}=\psi_{t}^{-}, \quad \nabla_{\mathrm{tg}} \psi^{+}=\nabla_{\mathrm{tg}} \psi^{-},
$$

for $\nabla_{\mathrm{tg}} \psi:=\nabla \psi-\left(\partial_{n} \psi\right) n$, the tangential component of the gradient on the boundary. In fact, since $\psi_{+}=\psi_{-}$ on $\tilde{\Sigma}$, as the tangential derivative just depends on the image of the function on the boundary, it holds that $\nabla_{\mathrm{tg}} \psi^{+}=\nabla_{\mathrm{tg}} \psi^{-}$. In all the terms of (3.12) we can omit the sign when we are working on $\tilde{\Sigma}$ to make the proof clearer.

Next, we consider the equality:

$$
L_{1}^{ \pm} \psi^{ \pm}+L_{2}^{ \pm} \psi^{ \pm}=L_{3}^{ \pm} \psi^{ \pm}
$$

for:

$$
\left\{\begin{array}{l}
L_{1}^{ \pm} \psi^{ \pm}:=-2 \varepsilon s \tau^{2}|\nabla \eta|^{2} \xi_{ \pm} \psi^{ \pm} \mp 2 \varepsilon s \tau \xi_{ \pm} \nabla \eta \cdot \nabla \psi^{ \pm}+\psi_{t}^{ \pm} \\
L_{2}^{ \pm} \psi^{ \pm}:=\varepsilon s^{2} \tau^{2}|\nabla \eta|^{2} \xi_{ \pm}^{2} \psi^{ \pm}+\varepsilon \Delta \psi^{ \pm}+s \partial_{t}\left(\alpha_{ \pm}\right) \psi^{ \pm}-\frac{1}{4 \varepsilon} \psi^{ \pm} \\
L_{3}^{ \pm} \psi^{ \pm}:= \pm \varepsilon s \tau \Delta \eta \xi_{ \pm} \psi^{ \pm}-\varepsilon s \tau^{2}|\nabla \eta|^{2} \xi_{ \pm} \psi^{ \pm}
\end{array}\right.
$$

As usual, we denote $\left(L_{i}^{ \pm} \psi\right)_{j}$ the $j$ th summand of $L_{i}^{ \pm} \psi$. Moreover, we calculate the product $\left(L_{1}^{ \pm} \psi^{ \pm}, L_{2}^{ \pm} \psi^{ \pm}\right)_{L^{2}(\tilde{Q})}$. To do so, we first compute it for generic functions $\phi \in C^{\infty}(\bar{Q})$ and then use a density argument.

To begin with, we have for $\tau \geq C$ and $s \geq C \tilde{T}^{2}$ that (see (3.7) for the definition of $\delta$ ):

$$
\left(\left(L_{1}^{ \pm} \psi^{ \pm}\right)_{1}+\left(L_{1}^{ \pm} \psi^{ \pm}\right)_{2},\left(L_{2}^{ \pm} \psi^{ \pm}\right)_{1}\right)_{L^{2}(\tilde{Q})}
$$




$$
\begin{aligned}
& =\varepsilon^{2} s^{3} \tau^{4} \iint_{\tilde{Q}}|\nabla \eta|^{4} \xi_{ \pm}^{3}\left|\psi^{ \pm}\right|^{2}+O\left(\varepsilon^{2} s^{2} \tau^{3} \iint_{\tilde{Q}} \xi_{ \pm}^{3}\left|\psi^{ \pm}\right|^{2}\right) \mp \varepsilon^{2} s^{3} \tau^{3} \iint_{\tilde{\Sigma}}|\nabla \eta|^{2} \xi^{3} \partial_{n} \eta|\psi|^{2} \\
& \geq \frac{3 \delta^{4}}{4} \varepsilon^{2} s^{3} \tau^{4} \iint_{\tilde{Q}} \xi_{ \pm}^{3}\left|\psi^{ \pm}\right|^{2}-\delta^{4} \varepsilon^{2} s^{3} \tau^{4} \iint_{\tilde{Q}_{\omega_{0}}} \xi_{ \pm}^{3}\left|\psi^{ \pm}\right|^{2} \mp \varepsilon^{2} s^{3} \tau^{3} \iint_{\tilde{\Sigma}}|\nabla \eta|^{2} \xi^{3} \partial_{n} \eta|\psi|^{2} .
\end{aligned}
$$

As for the third term in $L_{1}^{ \pm} \psi^{ \pm}$, we can integrate by parts in time and use the second inequality of (3.9) to obtain:

$$
\left(\left(L_{1}^{ \pm} \psi^{ \pm}\right)_{3},\left(L_{2}^{ \pm} \psi^{ \pm}\right)_{1}\right)_{L^{2}(\tilde{Q})}=O\left(\varepsilon \tilde{T} s^{2} \tau^{2} \iint_{\tilde{Q}} \xi_{ \pm}^{3}\left|\psi^{ \pm}\right|^{2}\right)
$$

Summing up, thanks to (3.15), (3.16) and (3.7) we obtain for $\tau \geq C$ and $s \geq C\left(\tilde{T}+\tilde{T}^{2}\right) \varepsilon^{-1}$ the estimate:

$$
\sum_{i \in\{+,-\}}\left(L_{1}^{i} \psi^{i},\left(L_{2}^{i} \psi^{i}\right)_{1}\right)_{L^{2}(\tilde{Q})} \geq \sum_{i \in\{+,-\}} \frac{\delta^{4}}{2} \varepsilon^{2} s^{3} \tau^{4} \iint_{\tilde{Q}} \xi_{i}^{3}\left|\psi^{i}\right|^{2}-\delta^{4} \varepsilon^{2} s^{3} \tau^{4} \iint_{\tilde{Q}_{\omega_{0}}} \xi_{i}^{3}\left|\psi^{i}\right|^{2} .
$$

To continue with, we find integrating by parts and with the Cauchy-Schwarz inequality that:

$$
\begin{aligned}
\left(\left(L_{1}^{ \pm} \psi^{ \pm}\right)_{1},\left(L_{2}^{ \pm} \psi^{ \pm}\right)_{2}\right)_{L^{2}(\tilde{Q})}=2 \varepsilon^{2} s \tau^{2} \iint_{\tilde{Q}}|\nabla \eta|^{2} \xi_{ \pm}\left|\nabla \psi^{ \pm}\right|^{2}-2 \varepsilon^{2} s \tau^{2} \iint_{\tilde{\Sigma}}|\nabla \eta|^{2} \xi\left(\partial_{n} \psi^{ \pm}\right) \psi \\
+O\left(\varepsilon^{2} s^{2} \tau^{4} \iint_{\tilde{Q}} \xi_{ \pm}^{2}\left|\psi^{ \pm}\right|^{2}+\varepsilon^{2} \tau^{2} \iint_{\tilde{Q}}\left|\nabla \psi^{ \pm}\right|^{2}\right)
\end{aligned}
$$

Next, integrating by parts and considering that $\eta=0$ on $\partial \Omega$ we obtain that:

$$
\begin{aligned}
\left(\left(L_{1}^{ \pm} \psi^{ \pm}\right)_{2},\right. & \left.\left(L_{2}^{ \pm} \psi^{ \pm}\right)_{2}\right)_{L^{2}(\tilde{Q})}=\mp 2 \varepsilon^{2} s \tau \iint_{\tilde{\Sigma}} \partial_{n} \eta \xi\left|\partial_{n} \psi^{ \pm}\right|^{2} \\
& +O\left(\varepsilon^{2} s \tau \iint_{\tilde{Q}} \xi_{ \pm}\left|\nabla \psi^{ \pm}\right|^{2}\right)+2 \varepsilon^{2} s \tau^{2} \iint_{\tilde{Q}} \xi_{ \pm}\left|\nabla \eta \cdot \nabla \psi^{ \pm}\right|^{2} \pm \varepsilon^{2} s \tau \iint_{\tilde{Q}} \xi_{ \pm} \nabla \eta \cdot \nabla\left|\nabla \psi^{ \pm}\right|^{2} \\
& =\mp 2 \varepsilon^{2} s \tau \iint_{\tilde{\Sigma}} \partial_{n} \eta \xi\left|\partial_{n} \psi^{ \pm}\right|^{2}+O\left(\varepsilon^{2} s \tau \iint_{\tilde{Q}} \xi_{ \pm}\left|\nabla \psi^{ \pm}\right|^{2}\right)+2 \varepsilon^{2} s \tau^{2} \iint_{\tilde{Q}} \xi_{ \pm}\left|\nabla \eta \cdot \nabla \psi^{ \pm}\right|^{2} \\
- & \varepsilon^{2} s \tau^{2} \iint_{\tilde{Q}}|\nabla \eta|^{2} \xi_{ \pm}\left|\nabla \psi^{ \pm}\right|^{2}+O\left(\varepsilon^{2} s \tau \iint_{\tilde{Q}} \xi_{ \pm}\left|\nabla \psi^{ \pm}\right|^{2}\right) \pm \varepsilon^{2} s \tau \iint_{\tilde{\Sigma}} \partial_{n} \eta \xi\left(\left|\nabla_{\mathrm{tg}} \psi\right|^{2}+\left|\partial_{n} \psi^{ \pm}\right|^{2}\right) .
\end{aligned}
$$

Moreover, we have that:

$$
\left(\left(L_{1}^{ \pm} \psi^{ \pm}\right)_{3},\left(L_{2}^{ \pm} \psi^{ \pm}\right)_{2}\right)_{L^{2}(\tilde{Q})}=\varepsilon \iint_{\tilde{\Sigma}} \psi_{t} \partial_{n} \psi^{ \pm}
$$

as $\psi(0, \cdot)=\psi(\tilde{T}, \cdot)=0$.

Let us compute the boundary terms involving $\partial_{n} \psi^{ \pm}$that appear in (3.18)-(3.20). On the one hand, from (3.11) we find that:

$$
-2 \varepsilon^{2} s \tau^{2} \iint_{\tilde{\Sigma}}|\nabla \eta|^{2} \xi\left(\partial_{n} \psi^{ \pm}\right) \psi=\mp 2 \varepsilon^{2} s^{2} \tau^{3} \iint_{\tilde{\Sigma}}|\nabla \eta|^{2} \partial_{n} \eta \xi^{2}|\psi|^{2}
$$




$$
-2 \varepsilon^{2} s \tau^{2} \int_{0}^{T}\left\langle\partial_{n} \phi,|\nabla \eta|^{2} \xi e^{-2 s \alpha} \phi\right\rangle_{H^{-1 / 2}(\partial \Omega), H^{1 / 2}(\partial \Omega)} .
$$

In addition, we obtain from $\eta=0$ on $\partial \Omega$ that:

$$
\begin{gathered}
\mp \varepsilon^{2} s \tau \iint_{\tilde{\Sigma}} \partial_{n} \eta \xi\left|\partial_{n} \psi^{ \pm}\right|^{2}=-2 \varepsilon^{2} s^{2} \tau^{2} \int_{0}^{T}\left\langle\partial_{n} \phi, e^{-2 s \alpha}|\nabla \eta|^{2} \xi^{2} \phi\right\rangle_{H^{-1 / 2}(\partial \Omega), H^{1 / 2}(\partial \Omega)} \\
\mp \varepsilon^{2} s^{3} \tau^{3} \iint_{\tilde{\Sigma}}\left(\partial_{n} \eta\right)^{3} \xi^{3}|\psi|^{2} \mp \varepsilon^{2} s \tau \iint_{\tilde{\Sigma}} \partial_{n} \eta \xi e^{-2 s \alpha}\left|\partial_{n} \phi\right|^{2} .
\end{gathered}
$$

Finally, we have that:

$$
\varepsilon \iint_{\tilde{\Sigma}} \psi_{t} \partial_{n} \psi^{ \pm}= \pm \varepsilon s \tau \iint_{\tilde{\Sigma}} \partial_{n} \eta \xi \psi \psi_{t}+\varepsilon \int_{0}^{T}\left\langle\partial_{n} \phi, e^{-s \alpha} \psi_{t}\right\rangle_{H^{-1 / 2}(\partial \Omega), H^{1 / 2}(\partial \Omega)} .
$$

Summing up, if we consider (3.18)-(3.23) we find that:

$$
\begin{aligned}
\sum_{i \in\{+,-\}}\left(L_{1}^{i} \psi^{i},\left(L_{2}^{i} \psi^{i}\right)_{2}\right)_{L^{2}(\tilde{Q})} & \geq \sum_{i \in\{+,-\}} O\left(\varepsilon^{2} s^{2} \tau^{4} \iint_{\tilde{Q}} \xi_{i}^{2}\left|\psi^{i}\right|^{2}\right) \\
& +\sum_{i \in\{+,-\}} \frac{\delta^{2}}{2} \varepsilon^{2} s \tau^{2} \iint_{\tilde{Q}} \xi_{i}\left|\nabla \psi^{i}\right|^{2}-\delta^{2} \varepsilon^{2} s \tau^{2} \iint_{\tilde{Q}_{\omega_{0}}} \xi_{i}\left|\nabla \psi^{i}\right|^{2} \\
& -4 \varepsilon^{2} \tau^{2} \int_{0}^{T}\left\langle\partial_{n} \phi, e^{-2 s \alpha}|\nabla \eta|^{2}\left(s \xi+s^{2} \xi^{2}\right) \phi\right\rangle_{H^{-1 / 2}(\partial \Omega), H^{1 / 2}(\partial \Omega)} \\
& +2 \varepsilon \int_{0}^{T}\left\langle\partial_{n} \phi, e^{-s \alpha} \psi_{t}\right\rangle_{H^{-1 / 2}(\partial \Omega), H^{1 / 2}(\partial \Omega)} .
\end{aligned}
$$

Next, we obtain from the second equation of (3.9) that:

$$
\left(\left(L_{1}^{ \pm} \psi^{ \pm}\right)_{1},\left(L_{2}^{ \pm} \psi^{ \pm}\right)_{3}\right)_{L^{2}(\tilde{Q})}=O\left(\varepsilon s^{2} \tau^{2} \tilde{T} \iint_{\tilde{Q}} \xi_{ \pm}^{3}\left|\psi^{ \pm}\right|^{2}\right)
$$

In addition, integrating by parts we find that:

$$
\left(\left(L_{1}^{ \pm} \psi^{ \pm}\right)_{2},\left(L_{2}^{ \pm} \psi^{ \pm}\right)_{3}\right)_{L^{2}(\tilde{Q})}=O\left(\varepsilon s^{2} \tau^{2} \tilde{T} \iint_{\tilde{Q}} \xi_{ \pm}^{3}\left|\psi^{ \pm}\right|^{2}\right) \mp \varepsilon s^{2} \tau \iint_{\tilde{\Sigma}} \xi \partial_{t} \alpha \partial_{n} \eta|\psi|^{2} .
$$

Finally, from the third equation of (3.9) we find that:

$$
\left(\left(L_{1}^{ \pm} \psi^{ \pm}\right)_{3},\left(L_{2}^{ \pm} \psi^{ \pm}\right)_{3}\right)_{L^{2}(\tilde{Q})}=-\frac{s}{2} \iint_{\tilde{Q}} \partial_{t t}\left(\alpha_{ \pm}\right)\left|\psi^{ \pm}\right|^{2}=O\left(s \iint_{\tilde{Q}} \xi_{ \pm}^{2}\left(1+\tilde{T}^{2} \xi_{ \pm}\right)\left|\psi^{ \pm}\right|^{2}\right)
$$

Summing up, combining (3.25)-(3.27) we obtain that:

$$
\sum_{i \in\{+,-\}}\left(L_{1}^{i} \psi^{i},\left(L_{2}^{i} \psi^{i}\right)_{3}\right)_{L^{2}(\tilde{Q})}=O\left(\varepsilon^{2} s^{3} \tau^{2} \iint_{\tilde{Q}} \xi_{ \pm}^{3}\left|\psi^{ \pm}\right|^{2}\right) .
$$


To continue with, we have that:

$$
\left(\left(L_{1}^{ \pm} \psi^{ \pm}\right)_{1},\left(L_{2}^{ \pm} \psi^{ \pm}\right)_{4}\right)_{L^{2}(\tilde{Q})}=O\left(s \tau^{2} \iint_{\tilde{Q}} \xi_{ \pm}\left|\psi^{ \pm}\right|^{2}\right)
$$

Next, integrating by parts we obtain that:

$$
\left(\left(L_{1}^{ \pm} \psi^{ \pm}\right)_{2},\left(L_{2}^{ \pm} \psi^{ \pm}\right)_{4}\right)_{L^{2}(\tilde{Q})}=O\left(s \tau^{2} \iint_{\tilde{Q}} \xi_{ \pm}\left|\psi^{ \pm}\right|^{2}\right) \pm \frac{s \tau}{4} \iint_{\tilde{\Sigma}} \xi \partial_{n} \eta|\psi|^{2}
$$

Finally, we obtain from $\psi^{ \pm}(0, \cdot)=\psi^{ \pm}(T, \cdot)=0$ that:

$$
\left(\left(L_{1}^{ \pm} \psi^{ \pm}\right)_{3},\left(L_{2}^{ \pm} \psi^{ \pm}\right)_{4}\right)_{L^{2}(\tilde{Q})}=0
$$

Summing up the results obtained in (3.29)-(3.31), we obtain that:

$$
\sum_{i \in\{+,-\}}\left(L_{1}^{i} \psi^{i},\left(L_{2}^{i} \psi^{i}\right)_{4}\right)_{L^{2}(\tilde{Q})}=O\left(s \tau^{2} \iint_{\tilde{Q}} \xi_{ \pm}\left|\psi^{ \pm}\right|^{2}\right)
$$

So, if we add (3.17), (3.24), (3.28) and (3.32), we find, after absorptions, for $\tau \geq C$ and $s \geq C\left(\tilde{T}+\tilde{T}^{2}\right) \varepsilon^{-1}$ :

$$
\begin{aligned}
2 \sum_{i \in\{+,-\}}\left(L_{1}^{i} \psi^{i}, L_{2}^{i} \psi^{i}\right) & +2 \delta^{4} \varepsilon^{2} s^{3} \tau^{4} \iint_{\tilde{Q}_{\omega_{0}}} \xi_{i}^{3}\left|\psi^{i}\right|^{2}+2 \delta^{2} \varepsilon^{2} s \tau^{2} \iint_{\tilde{Q}_{\omega_{0}}} \xi_{i}\left|\nabla \psi^{i}\right|^{2} \\
& \geq \sum_{i \in\{+,-\}} \frac{\delta^{4}}{4} \varepsilon^{2} s^{3} \tau^{4} \iint_{\tilde{Q}} \xi_{i}^{3}\left|\psi^{i}\right|^{2}+\frac{\delta^{2}}{2} \varepsilon^{2} s \tau^{2} \iint_{\tilde{Q}} \xi_{i}\left|\nabla \psi^{i}\right|^{2} \\
& -4 \varepsilon^{2} \tau^{2} \int_{0}^{T}\left\langle\partial_{n} \phi, e^{-2 s \alpha}|\nabla \eta|^{2}\left(s \xi+s^{2} \xi^{2}\right) \phi\right\rangle_{H^{-1 / 2}(\partial \Omega), H^{1 / 2}(\partial \Omega)} \\
& +2 \varepsilon \int_{0}^{T}\left\langle\partial_{n} \phi, e^{-s \alpha} \psi_{t}\right\rangle_{H^{-1 / 2}(\partial \Omega), H^{1 / 2}(\partial \Omega)} .
\end{aligned}
$$

Using the density of $C^{\infty}(\bar{Q})$ in $H^{1}\left(0, T ; \mathcal{D}_{\varepsilon}\right)$ we obtain that (3.33) is true for all solutions of (2.4) such that $\phi^{\tilde{T}} \in \mathcal{D}(\Omega)$. So, from now on $\phi$ denotes any solution of $(2.4)$ with initial value in $\mathcal{D}(\Omega)$.

Considering the second and third equations of (2.4), that $a^{\varepsilon}$ is independent of the time variable and that $\psi^{ \pm}(0, \cdot)=\psi^{ \pm}(\tilde{T}, \cdot)=0$ we find that:

$$
\begin{aligned}
& -4 \varepsilon^{2} \tau^{2} \int_{0}^{T}\left\langle\partial_{n} \phi, e^{-2 s \alpha}|\nabla \eta|^{2}\left(s \xi+s^{2} \xi^{2}\right) \phi\right\rangle_{H^{-1 / 2}(\partial \Omega), H^{1 / 2}(\partial \Omega)} \\
& +2 \varepsilon \int_{0}^{T}\left\langle\partial_{n} \phi, e^{-s \alpha} \psi_{t}\right\rangle_{H^{-1 / 2}(\partial \Omega), H^{1 / 2}(\partial \Omega)} \\
& =4 \varepsilon^{2} \tau^{2} \iint_{\tilde{\Sigma}_{N}}|\nabla \eta|^{2}\left(s \xi+s^{2} \xi^{2}\right)\left(a^{\varepsilon}+\frac{n_{1}}{2 \varepsilon}\right)|\psi|^{2}+2 \varepsilon \iint_{\tilde{\Sigma}_{N}}\left(a^{\varepsilon}+\frac{n_{1}}{2 \varepsilon}\right) \psi \psi_{t} \\
& =4 \varepsilon^{2} \tau^{2} \iint_{\tilde{\Sigma}_{N}}|\nabla \eta|^{2}\left(s \xi+s^{2} \xi^{2}\right)\left(a^{\varepsilon}+\frac{n_{1}}{2 \varepsilon}\right)|\psi|^{2} .
\end{aligned}
$$


Consequently, from (3.33) and (3.34) we obtain for all solutions of (2.4) such that $\phi^{\tilde{T}} \in \mathcal{D}(\Omega)$ the estimate:

$$
\begin{aligned}
2 \sum_{i \in\{+,-\}}\left(L_{1}^{i} \psi^{i}, L_{2}^{i} \psi^{i}\right) & +2 \delta^{4} \varepsilon^{2} s^{3} \tau^{4} \iint_{\tilde{Q}_{\omega_{0}}} \xi_{i}^{3}\left|\psi^{i}\right|^{2}+2 \delta^{2} \varepsilon^{2} s \tau^{2} \iint_{\tilde{Q}_{\omega_{0}}} \xi_{i}\left|\nabla \psi^{i}\right|^{2} \\
& \geq \sum_{i \in\{+,-\}} \frac{\delta^{4}}{2} \varepsilon^{2} s^{3} \tau^{4} \iint_{\tilde{Q}} \xi_{i}^{3}\left|\psi^{i}\right|^{2}+\frac{\delta^{2}}{2} \varepsilon^{2} s \tau^{2} \iint_{\tilde{Q}} \xi_{i}\left|\nabla \psi^{i}\right|^{2} \\
& +4 \varepsilon^{2} \tau^{2} \iint_{\tilde{\Sigma}_{N}}|\nabla \eta|^{2}\left(s \xi+s^{2} \xi^{2}\right)\left(a^{\varepsilon}+\frac{n_{1}}{2 \varepsilon}\right)|\psi|^{2}
\end{aligned}
$$

We recall that $\inf _{\partial \Omega}|\nabla \eta|>0$ thanks to (3.7). Thus, using (2.9) it is classical to obtain (3.10) from (3.35) (see, for instance, [19] and [17]) for all solutions of (2.4) such that $\phi^{\tilde{T}} \in \mathcal{D}(\Omega)$. Afterwards, we obtain (3.10) for all solutions of (2.4) such that $\phi^{\tilde{T}} \in L^{2}(\Omega)$ by density.

Remark 3.6. The trace term in (3.10) can be absorbed by considering $\tau>C\left(\varepsilon^{-1}+\left\|a^{\varepsilon}\right\|_{L^{\infty}(\Gamma)}\right)$. Indeed, in that case we have that:

$$
s^{2} \tau^{2} \iint_{\tilde{\Sigma}_{N}} e^{-2 s \alpha_{+}} \xi_{+}^{2}\left|a^{\varepsilon}+\frac{n_{1}}{2 \varepsilon}\right||\phi|^{2} \leq C\left(s^{3} \tau^{3} \iint_{\tilde{Q}} e^{-2 s \alpha_{+}} \xi_{+}^{3}|\phi|^{2}+s \tau^{2} \iint_{\tilde{Q}} e^{-2 s \alpha_{+}} \xi_{+}|\nabla \phi|^{2}\right),
$$

a term which can be absorbed for $\tau>C$. This allows to prove the null observability of (2.4), which implies the null observability of (2.1) and consequently the null controllability of (1.1), but with an estimate of the cost of the control of $\exp \left(e^{C\left(\varepsilon^{-1}+\left\|a^{\varepsilon}\right\|_{L}(\Gamma)\right)}\right)$.

\subsection{Conclusion of the proof of Theorem 2.7}

Let $T \geq 1$. Considering Proposition 3.5 for $\tilde{T}=1$ and Remark 2.1 we obtain for all $\varphi^{T} \in L^{2}(\Omega), \tau \geq C$ and $s \geq C \varepsilon^{-1}$ the estimate:

$$
\begin{aligned}
s^{3} \tau^{4} \int_{0}^{1} \int_{\Omega} e^{-2 s \alpha_{+}(t, x)+\varepsilon^{-1} x_{1}} \xi_{+}^{3}(t, x) \mid \varphi & \left.(T-1+t, x)\right|^{2} \mathrm{~d} x \mathrm{~d} t \\
& \leq C s^{3} \tau^{4} \int_{0}^{1} \int_{\omega} e^{-2 s \alpha_{+}(t, x)+\varepsilon^{-1} x_{1}} \xi_{+}^{3}(t, x)|\varphi(T-1+t, x)|^{2} \mathrm{~d} x \mathrm{~d} t,
\end{aligned}
$$

for $\varphi$ the solution of (2.1). So, fixing $\tau$ large enough and $s=s_{0} \varepsilon^{-1}$ for $s_{0}$ large enough, we have that (3.36) implies that:

$$
\|\varphi\|_{L^{2}((T-2 / 3, T-1 / 3) \times \Omega)} \leq C e^{C \varepsilon^{-1}}\|\varphi\|_{L^{2}((T-1, T) \times \omega)} \leq C e^{C \varepsilon^{-1}}\|\varphi\|_{L^{2}\left(Q_{\omega}\right)} .
$$

Moreover, from Lemma 3.3 taking $T_{0}=\delta=1$ we find a constant $C>0$ such that for all $t^{\prime} \in(T-2 / 3, T-1 / 3)$ we have that:

$$
\|\varphi(0, \cdot)\|_{L^{2}(\Omega)} \leq C \exp \left[\frac{2 \operatorname{diam}_{x_{1}}(\Omega)+1-t^{\prime}}{4 \varepsilon}\right]\left\|\varphi\left(t^{\prime}, \cdot\right)\right\|_{L^{2}(\Omega)} \leq C \exp \left[\frac{C-T}{4 \varepsilon}\right]\left\|\varphi\left(t^{\prime}, \cdot\right)\right\|_{L^{2}(\Omega)} .
$$

So, combining (3.37) and (3.38), we obtain that:

$$
\|\varphi(0, \cdot)\|_{L^{2}(\Omega)} \leq C \exp \left[\frac{C-T}{4 \varepsilon}\right]\|\varphi\|_{L^{2}((T-2 / 3, T-1 / 3) \times \Omega)} \leq C \exp \left[\frac{C-T}{4 \varepsilon}\right]\|\varphi\|_{L^{2}\left(Q_{\omega}\right)} .
$$


In particular, if $T$ is sufficiently large, from (2.2) and (3.39) we obtain (2.10).

\section{Some CONTROLlability PROBlems in Which The COST OF THE CONTROL EXPLODES EXPONENTIALLY}

In this section we study some control problems in which (2.11) is satisfied. In particular, in Section 4.1 we prove Proposition 2.10, in Section 4.2 we prove Theorem 2.8 and in Section 4.3 we prove Theorem 2.9.

\subsection{Proof of Proposition 2.10}

Let $v \in H^{1}(\Omega)$ satisfying $\|v\|_{L^{2}(\Omega)}=1$ and $v_{\mid \Gamma^{*}}=0$. We find from the Green formula and a weighted Cauchy-Schwarz inequality that:

$$
\int_{\Gamma} \frac{n_{1}}{2 \varepsilon}|v|^{2} \mathrm{~d} x=\int_{\partial \Omega} \frac{n_{1}}{2 \varepsilon}|v|^{2} \mathrm{~d} x=\int_{\Omega} \frac{v \partial_{x_{1}} v}{\varepsilon} \mathrm{d} x \geq-\int_{\Omega}\left|\partial_{x_{1}} v\right|^{2} \mathrm{~d} x-\frac{1}{4 \varepsilon^{2}} \int_{\Omega}|v|^{2} \mathrm{~d} x .
$$

Thus, from $a^{\varepsilon} \geq 0, v \in H^{1}(\Omega),\|v\|_{L^{2}(\Omega)}=1$ and $v_{\mid \Gamma^{*}}=0$ on $\Gamma^{*}$ we find that:

$$
\int_{\Omega}|\nabla v|^{2} \mathrm{~d} x+\int_{\Gamma}\left(a^{\varepsilon}+\frac{n_{1}}{2 \varepsilon}\right)|v|^{2} \mathrm{~d} x \geq \int_{\Omega}|\nabla v|^{2} \mathrm{~d} x-\int_{\Omega}\left|\partial_{x_{1}} v\right|^{2} \mathrm{~d} x-\frac{1}{4 \varepsilon^{2}} \geq-\frac{1}{4 \varepsilon^{2}}
$$

Finally, we obtain (2.18) from Remark 2.2, (2.8) and (4.1).

In order to have an equality in (4.1) we need that $a^{\varepsilon} v=0$, that $v$ just depends on $x_{1}$ and that $\partial_{x_{1}} v=-(2 \varepsilon)^{-1} v ;$ i.e. we need that $v=\frac{e^{-(2 \varepsilon)^{-1} x_{1}}}{\left\|e^{-(2 \varepsilon)^{-1} x_{1}}\right\|_{L^{2}(\Omega)}}$, and thus we also need $\Gamma=\partial \Omega, a^{\varepsilon}=0$ and $u^{\varepsilon}=\frac{e^{-\varepsilon^{-1} x_{1}}}{\left\|e^{-(2 \varepsilon)^{-1} x_{1}}\right\|_{L^{2}(\Omega)}}$ (this last one by Remark 2.2).

\subsection{Proof of Theorem 2.8}

We recall that in this section we are studying the case in which we have pure Neumann boundary conditions; that is, (1.1) with $a^{\varepsilon}=0$ and $\Gamma=\partial \Omega$. In order to prove (2.15) we consider the adjoint system of (2.12), which is given by (2.1). In particular, since $a^{\varepsilon}=0$ and $\Gamma=\partial \Omega,(2.1)$ becomes:

$$
\begin{cases}-\varphi_{t}-\varepsilon \Delta \varphi-\partial_{x_{1}} \varphi=0, & \text { in } Q, \\ \varepsilon \partial_{n} \varphi+n_{1} \varphi=0, & \text { on } \Sigma, \\ \varphi(T, \cdot)=\varphi^{T}, & \text { on } \Omega .\end{cases}
$$

To prove Theorem 2.8 we use (2.2) for $\bar{\varphi}(x)=e^{-x_{1} \varepsilon^{-1}}$, which clearly is a (steady) solution of (4.2). On the one hand, we remark that (see (2.13) for the notation):

$$
\|\bar{\varphi}(0, \cdot)\|_{L^{2}(\Omega)} \geq\left|\pi_{1}^{-1}\left(p_{l}, p_{l}+h / 2\right) \cap \Omega\right|^{1 / 2} \exp \left(\frac{-p_{l}-h / 2}{\varepsilon}\right) .
$$

Because $\Omega$ is an open set and because of (2.13) we have that:

$$
\left|\pi_{1}^{-1}\left(p_{l}, p_{l}+h / 2\right) \cap \Omega\right|>0 .
$$


On the other hand, (2.14) implies the estimate:

$$
\|\bar{\varphi}\|_{L^{2}\left(Q_{\omega}\right)} \leq T^{1 / 2}|\omega|^{1 / 2}\left\|e^{-x_{1} \varepsilon^{-1}}\right\|_{L^{\infty}\left(\pi_{1}^{-1}\left(p_{l}+h, p_{r}\right) \cap \omega\right)} \leq T^{1 / 2}|\omega|^{1 / 2} \exp \left(\frac{-p_{l}-h}{\varepsilon}\right) .
$$

Consequently, combining (2.2), (4.3) and (4.4) we find that:

$$
K(\Omega, \omega, T, \varepsilon) \geq \frac{\|\bar{\varphi}(0, \cdot)\|_{L^{2}(\Omega)}}{\|\bar{\varphi}\|_{L^{2}\left(Q_{\omega}\right)}} \geq \frac{\left|\pi_{1}^{-1}\left(p_{l}, p_{l}+h / 2\right) \cap \Omega\right|^{1 / 2}}{T^{1 / 2}|\omega|^{1 / 2}} \exp \left(\frac{h}{2 \varepsilon}\right),
$$

which implies (2.15).

\subsection{Proof of Theorem 2.9}

We first remark that the adjoint system of (2.16) is given by (see (2.1)):

$$
\begin{cases}-\varphi_{t}-\varepsilon \partial_{x x} \varphi-\partial_{x} \varphi=0, & \text { in } Q, \\ \varepsilon \partial_{x} \varphi(\cdot,-L)+\varphi(\cdot,-L)=0, & \text { on }(0, T), \\ \varphi(\cdot, 0)=0, & \text { on }(0, T), \\ \varphi(T, \cdot)=\varphi^{T}, & \text { on }(-L, 0)\end{cases}
$$

In addition, the spectral problem (2.6) can be written as:

$$
\left\{\begin{array}{l}
-v^{\prime \prime}=\tilde{\lambda} v, \\
2 \varepsilon v^{\prime}(-L)+v(-L)=0, \\
v(0)=0 .
\end{array}\right.
$$

In order to prove Theorem 2.9, we first prove the following lemma:

Lemma 4.1. Let $L>0$. Then, for $\varepsilon$ small enough there is a unique $r_{\varepsilon}>0$ such that there are non-trivial solutions of (4.6) for $\tilde{\lambda}^{\varepsilon}=-r_{\varepsilon}^{2}$. In fact, we have:

$$
r_{\varepsilon} \in\left(0, \frac{1}{2 \varepsilon}\right)
$$

and the non-trivial solutions are those proportional to $\sinh \left(-r_{\varepsilon} x\right)$. In addition, we have the limit:

$$
\lim _{\varepsilon \rightarrow 0} \frac{1}{2 \varepsilon}-r_{\varepsilon}=0
$$

Remark 4.2. From Proposition 2.10 and Remark 2.2 we already know that all the eigenvalues are strictly bigger than $-(2 \varepsilon)^{-2}$; thus, it is clear that $r_{\varepsilon}$ must satisfy (4.7).

Proof of Lemma 4.1. The structure of the proof is the following: first we give an equivalent condition for $-r_{\varepsilon}^{2}$ to be an eigenvalue of (4.6), second we show that (4.6) has a unique strictly negative eigenvalue, and thirdly we prove (4.8).

Step 1: an equivalent condition. First, we recall that when $\tilde{\lambda}=-r^{2}<0$, the solutions of the first equation in (4.6) are given by:

$$
A e^{r x}+B e^{-r x}: \quad A, B \in \mathbb{R} .
$$


Moreover, the boundary conditions of (4.6) are equivalent to $(A, B)$ satisfying:

$$
\left\{\begin{array}{l}
\left(2 \varepsilon r e^{-r L}+e^{-r L}\right) A+\left(-2 \varepsilon r e^{r L}+e^{r L}\right) B=0, \\
A+B=0 .
\end{array}\right.
$$

Thus, (4.9) has a non-trivial solution if and only if:

$$
e^{-r L}+2 \varepsilon r e^{-r L}=e^{r L}-2 \varepsilon r e^{r L},
$$

that is, if and only if:

$$
\frac{1+2 \varepsilon r}{1-2 \varepsilon r}=e^{2 r L}
$$

In addition, from the second equation of (4.9) we obtain that the associated eigenfunctions are those proportional to $\sinh \left(-r_{\varepsilon} x\right)$. Finally, from (4.10) we have that all the possible positive roots are in $\left(0,(2 \varepsilon)^{-1}\right)$, so we just have to prove existence and uniqueness in that interval.

Step 2: proof of the fact that (4.10) has a unique solution in $\left(0,(2 \varepsilon)^{-1}\right)$. Let us denote:

$$
g_{1}(r):=\frac{1+2 \varepsilon r}{1-2 \varepsilon r}=-1+\frac{2}{1-2 \varepsilon r}, \quad g_{2}(r):=e^{2 r L} .
$$

We have the equality:

$$
g_{1}(0)=g_{2}(0)=1
$$

In addition, the derivative of the functions are given by:

$$
g_{1}^{\prime}(r)=\frac{4 \varepsilon}{(1-2 \varepsilon r)^{2}}, \quad g_{2}^{\prime}(r):=2 L e^{2 r L} .
$$

Consequently, combining (4.11) and (4.12) we obtain a constant $c>0$ such that for $\varepsilon$ small enough, $g_{2}>g_{1}$ in $(0, c)$. Moreover, since $\lim _{r \uparrow(2 \varepsilon)^{-1}} g_{1}(r)=+\infty$ and $g_{2}\left((2 \varepsilon)^{-1}\right) \in \mathbb{R}$, we have at least one root of $(4.10)$ in $\left(0,(2 \varepsilon)^{-1}\right)$. In order to show the uniqueness of the root, we define:

$$
g_{3}(r):=\frac{g_{2}^{\prime}(r)}{g_{1}^{\prime}(r)}=\frac{L}{2 \varepsilon}(1-2 \varepsilon r)^{2} e^{2 r L} .
$$

Since in $\left(0,(2 \varepsilon)^{-1}\right)$ the function $g_{3}^{\prime}$ has the same sign as $2 L(1-2 \varepsilon r)-4 \varepsilon$, we have $g_{3}^{\prime}(r) \leq 0$ if and only if:

$$
r \geq \frac{2 L-4 \varepsilon}{4 L \varepsilon}=\frac{1}{2 \varepsilon}-\frac{1}{L}
$$

So, since $g_{3}(0)=\frac{L}{4 \varepsilon}$, for $\varepsilon$ small enough there is a unique:

$$
\bar{r}_{\varepsilon} \in\left(\frac{1}{2 \varepsilon}-\frac{1}{L}, \frac{1}{2 \varepsilon}\right)
$$

such that $g_{3}\left(\bar{r}_{\varepsilon}\right)=1, g_{3}>1$ in $\left[0, \bar{r}_{\varepsilon}\right]$ and $g_{3}$ is strictly decreasing in $\left[\bar{r}_{\varepsilon},(2 \varepsilon)^{-1}\right]$. This implies that $g_{2}^{\prime}(r)>g_{1}^{\prime}(r)$ in $\left[0, \bar{r}_{\varepsilon}\right)$ and $g_{2}^{\prime}(r)<g_{1}^{\prime}(r)$ in $\left(\bar{r}_{\varepsilon},(2 \varepsilon)^{-1}\right)$. Consequently, (4.10) has a unique root in $\left(0,(2 \varepsilon)^{-1}\right)$ which, to be more precise, belongs to $\left[\bar{r}_{\varepsilon},(2 \varepsilon)^{-1}\right]$ and which we denote from now on by $r_{\varepsilon}$. 
Step 3: proof of (4.8). In order to prove (4.8) we first consider that for $\varepsilon$ small enough:

$$
r_{\varepsilon} \geq \bar{r}_{\varepsilon} \geq \frac{1}{2 \varepsilon}-\frac{1}{L} \geq \frac{1}{4 \varepsilon} .
$$

Moreover, from $g_{1}\left(r_{\varepsilon}\right)=g_{2}\left(r_{\varepsilon}\right)$ we obtain that:

$$
\frac{1 / \varepsilon}{\frac{1}{2 \varepsilon}-r_{\varepsilon}}=e^{2 r_{\varepsilon} L}+1
$$

which implies the equality:

$$
\frac{1}{2 \varepsilon}-r_{\varepsilon}=\frac{1}{\varepsilon\left(e^{2 r_{\varepsilon} L}+1\right)}
$$

Using (4.13), we find for $\varepsilon$ small enough that:

$$
\frac{1}{2 \varepsilon}-r_{\varepsilon} \leq \frac{1}{\varepsilon e^{L(2 \varepsilon)^{-1}}}
$$

Consequently, we obtain (4.8) from (4.14) and (4.7).

We now end the proof of Theorem 2.9. Remark 2.2 implies that:

$$
\left(u^{\varepsilon}(x), \lambda_{0}^{\varepsilon}\right):=\left(\sinh \left(-r_{\varepsilon} x\right) e^{-(2 \varepsilon)^{-1} x},-\varepsilon r_{\varepsilon}^{2}+\frac{1}{4 \varepsilon}\right),
$$

is a solution of (2.5). Hence $\hat{\varphi}(t, x)=u^{\varepsilon}(x) e^{\lambda_{0}^{\varepsilon}(t-T)}$ is a solution of (4.5). Moreover, we obtain from $\varepsilon r_{\varepsilon}<1 / 2$ (see (4.7)) and (4.8) the limit:

$$
\lim _{\varepsilon \rightarrow 0} \lambda_{0}^{\varepsilon}=\lim _{\varepsilon \rightarrow 0} \varepsilon\left(\frac{1}{2 \varepsilon}-r_{\varepsilon}\right)\left(\frac{1}{2 \varepsilon}+r_{\varepsilon}\right)=\lim _{\varepsilon \rightarrow 0}\left(\frac{1}{2 \varepsilon}-r_{\varepsilon}\right)\left(\frac{1}{2}+\varepsilon r_{\varepsilon}\right)=0 .
$$

We prove (2.17) with (2.2). On the one hand, for $x \in(-L,-L+h / 2)$ and $\varepsilon$ small enough we have the bound:

$$
u^{\varepsilon}(x)=\sinh \left(-r_{\varepsilon} x\right) e^{-(2 \varepsilon)^{-1} x} \geq \frac{1}{4} e^{-\left(r_{\varepsilon}+(2 \varepsilon)^{-1}\right) x} \geq \frac{1}{4} e^{\left(r_{\varepsilon}+(2 \varepsilon)^{-1}\right)(L-h / 2)} .
$$

Moreover, using (4.16) we find for $\varepsilon$ small enough that $e^{-\lambda_{0}^{\varepsilon} T} \geq 1 / 2$. So we obtain the bound:

$$
\|\hat{\varphi}(0, \cdot)\|_{L^{2}(-L, 0)} \geq\|\hat{\varphi}(0, \cdot)\|_{L^{2}(-L,-L+h / 2)}=e^{-\lambda_{0}^{\varepsilon} T}\left\|u^{\varepsilon}\right\|_{L^{2}(-L,-L+h / 2)} \geq \frac{\sqrt{2 h}}{16} e^{\left(r_{\varepsilon}+(2 \varepsilon)^{-1}\right)(L-h / 2)} .
$$

On the other hand, from $\omega \subset(-L+h, 0)$ we obtain the estimate:

$$
\|\hat{\varphi}\|_{L^{2}\left(Q_{\omega}\right)} \leq \sqrt{T(L-h)} e^{\left(r_{\varepsilon}+(2 \varepsilon)^{-1}\right)(L-h)} .
$$

To prove (4.18) we have used that:

$$
\left\|e^{\lambda_{0}^{\varepsilon}(t-T)}\right\|_{L^{\infty}(0, T)} \leq 1,
$$


and that in $(-L+h, 0)$ :

$$
\sinh \left(-r_{\varepsilon} x\right) e^{-(2 \varepsilon)^{-1} x} \leq \frac{e^{-\left(r_{\varepsilon}+(2 \varepsilon)^{-1}\right) x}}{2} \leq \frac{e^{\left(r_{\varepsilon}+(2 \varepsilon)^{-1}\right)(L-h)}}{2} .
$$

Finally, combining (2.2), (4.17), (4.18) and that $r_{\varepsilon} \geq 0$, we obtain (2.17).

Remark 4.3. The key idea of the proof is that $\lambda_{0}^{\varepsilon} \rightarrow 0$, but we also need to know how the eigenfunctions are distributed in $\Omega$.

\section{Cost of the CONTROL When $\omega=\Omega$}

In this section we study some control problems in which the control domain is $\Omega$. In particular, in Section 5.1 we prove Proposition 2.15, in Section 5.2 we prove Proposition 2.16, and in Section 5.3 we prove Proposition 2.17.

\subsection{Proof of Proposition 2.15}

From Definition 2.5 we obtain that $\varphi^{\varepsilon}(t, x)=u_{0}^{\varepsilon}(x) e^{\lambda_{0}^{\varepsilon}(T-t)}$ is a solution of (2.1). Furthermore, (2.19) implies that:

$$
\lim _{\varepsilon \rightarrow 0} \int_{0}^{T} e^{-2 \lambda_{0}^{\varepsilon}(T-t)} d t \rightarrow T
$$

Consequently, we have the limit:

$$
\lim _{\varepsilon \rightarrow 0} \frac{\left\|\varphi^{\varepsilon}(0, \cdot)\right\|_{L^{2}(\Omega)}^{2}}{\iint_{Q}\left|\varphi^{\varepsilon}\right|^{2} \mathrm{~d} x \mathrm{~d} t}=\lim _{\varepsilon \rightarrow 0} \frac{\left\|u_{0}^{\varepsilon} e^{-\lambda_{0}^{\varepsilon} T}\right\|_{L^{2}(\Omega)}^{2}}{\iint_{Q}\left|u_{0}^{\varepsilon} e^{-\lambda_{0}^{\varepsilon}(T-t)}\right|^{2} \mathrm{~d} x \mathrm{~d} t}=\lim _{\varepsilon \rightarrow 0} \frac{e^{-2 \lambda_{0}^{\varepsilon} T}}{\int_{0}^{T} e^{-2 \lambda_{0}^{\varepsilon}(T-t)} d t}=\frac{1}{T},
$$

which together with (2.2) implies (2.20).

\subsection{Proof of Proposition 2.16}

Let $\varphi$ be a solution of $(2.1)$ and $\chi \leq 1$ be a regular positive cut-off function whose value is 1 in $[0,1 / 3]$ and 0 in $[2 / 3,1]$. Then:

$$
\begin{aligned}
\frac{1}{2} \int_{\Omega}|\varphi(0, x)|^{2} \mathrm{~d} x= & -\frac{1}{2} \iint_{Q} \partial_{t}\left(\chi\left(\frac{t}{T}\right)|\varphi|^{2}\right) \mathrm{d} x \mathrm{~d} t \\
= & -\frac{1}{2 T} \iint_{Q} \chi^{\prime}\left(\frac{t}{T}\right)|\varphi|^{2} \mathrm{~d} x \mathrm{~d} t+\iint_{Q} \chi\left(\frac{t}{T}\right)\left(\varepsilon \Delta \varphi+\partial_{x_{1}} \varphi\right) \varphi \mathrm{d} x \mathrm{~d} t \\
= & -\frac{1}{2 T} \iint_{Q} \chi^{\prime}\left(\frac{t}{T}\right)|\varphi|^{2} \mathrm{~d} x \mathrm{~d} t-\varepsilon \iint_{Q} \chi\left(\frac{t}{T}\right)|\nabla \varphi|^{2} \mathrm{~d} x \mathrm{~d} t \\
& -\iint_{\Sigma_{N}} \chi\left(\frac{t}{T}\right)\left(a^{\varepsilon}+\frac{n_{1}}{2}\right)|\varphi|^{2} \mathrm{~d} x \mathrm{~d} t \leq C\left(\frac{1}{T}+\frac{1}{\varepsilon}\right) \iint_{Q}|\varphi|^{2} \mathrm{~d} x \mathrm{~d} t
\end{aligned}
$$

For the last inequality in (5.1) we use that $a^{\varepsilon} \geq 0$ and the following classical estimate:

$$
\left.\left.\left|\iint_{\Sigma_{N}} \chi\left(\frac{t}{T}\right) \frac{n_{1}}{2}\right| \varphi\right|^{2}\left|\mathrm{~d} x \mathrm{~d} t \leq \varepsilon \iint_{Q} \chi\left(\frac{t}{T}\right)\right| \nabla \varphi\right|^{2} \mathrm{~d} x \mathrm{~d} t+\frac{C}{\varepsilon} \iint_{Q}|\varphi|^{2} \mathrm{~d} x \mathrm{~d} t .
$$

Thereby, we obtain (2.21) from (2.2) and (5.1). 


\subsection{Proof of Proposition 2.17}

We prove Proposition 2.17 thanks to an explicit computation of the eigenvalues of (4.2). For that purpose, we remark that the spectral problem (2.6) when $\Gamma=\partial \Omega$ and $a^{\varepsilon}=0$ is given by:

$$
\begin{cases}-v^{\prime \prime}=\tilde{\lambda} v, & \text { in }(-L, 0) \\ 2 \varepsilon v^{\prime}+v=0, & \text { on }\{-L, 0\}\end{cases}
$$

It can be proved easily that the only negative eigenvalue is $\tilde{\lambda}_{0}^{\varepsilon}=-\frac{1}{4 \varepsilon^{2}}$ and that the associated eigenfunction is:

$$
v_{0}^{\varepsilon}(x)=\frac{e^{-(2 \varepsilon)^{-1} x}}{\left\|e^{-(2 \varepsilon)^{-1} z}\right\|_{L^{2}(\Omega, \mathrm{d} z)}} .
$$

In addition, it can be proved for $m \in \mathbb{N}_{*}$ that:

$$
\tilde{\lambda}_{m}^{\varepsilon}=\left(\frac{\pi m}{L}\right)^{2}
$$

and its associated eigenfunction is:

$$
v_{m}^{\varepsilon}(x)=\frac{-2 \varepsilon \sqrt{\tilde{\lambda}_{m}} \cos \left(\sqrt{\tilde{\lambda}_{m}} x\right)+\sin \left(\sqrt{\tilde{\lambda}_{m}} x\right)}{\left\|2 \varepsilon \sqrt{\tilde{\lambda}_{m}} \cos \left(\sqrt{\tilde{\lambda}_{m}} z\right)+\sin \left(\sqrt{\tilde{\lambda}_{m}} z\right)\right\|_{L^{2}(\Omega, \mathrm{d} z)}} .
$$

Consequently, Remark 2.2 implies that the spectral decomposition by the elliptic operator associated to the adjoint system is given by $u_{m}^{\varepsilon}(x)=v_{m}^{\varepsilon}(x) e^{-(2 \varepsilon)^{-1} x}$, and their associated eigenvalues are given by $\lambda_{m}^{\varepsilon}=\varepsilon \tilde{\lambda}_{m}+$ $(4 \varepsilon)^{-1}$. In particular, we have that:

$$
u_{0}^{\varepsilon}(x)=\frac{e^{-\varepsilon^{-1} x}}{\left\|e^{-(2 \varepsilon)^{-1} z}\right\|_{L^{2}(\Omega, \mathrm{d} z)}}, \quad \lambda_{0}^{\varepsilon}=0 .
$$

In addition, we have the following result:

Lemma 5.1. Let $L>0$ and $\Omega:=(-L, 0)$. Then, for all $\varphi^{T} \in L^{2}(\Omega)$ the solution of (4.2) satisfies in $C^{0}\left([0, T] ; L^{2}(\Omega)\right)$ :

$$
\begin{aligned}
& \varphi(t, x)= \frac{\int_{\Omega} \varphi^{T}(z) \mathrm{d} z}{\left\|e^{-(2 \varepsilon)^{-1} z}\right\|_{L^{2}(\Omega, \mathrm{d} z)}} e^{-\varepsilon^{-1} x} \\
& \quad+\sum_{m \in \mathbb{N}_{*}}\left(\int_{\Omega} \varphi^{T}(z) e^{(2 \varepsilon)^{-1} z} v_{m}^{\varepsilon}(z) \mathrm{d} z\right) v_{m}^{\varepsilon}(x) e^{-(2 \varepsilon)^{-1} x} \exp \left[-\left(\varepsilon \tilde{\lambda}_{m}^{\varepsilon}+\frac{1}{4 \varepsilon}\right)(T-t)\right] .
\end{aligned}
$$

In particular, the series in the right-hand side of (5.3) is absolutely convergent in $L^{2}(\Omega)$ for all $t<T$. Moreover, for all $T_{0}>0$ and $\delta>0$ there is $C>0$ such that for all $\varphi^{T} \in L^{2}(\Omega), T \geq T_{0}$ and $t \in\left[0, T-T_{0}\right]$ we have the estimate:

$$
\left\|\sum_{m \in \mathbb{N}_{*}}\left(\int_{\Omega} \varphi^{T}(z) e^{(2 \varepsilon)^{-1} z} v_{m}^{\varepsilon}(z) \mathrm{d} z\right) v_{m}^{\varepsilon}(x) e^{-(2 \varepsilon)^{-1} x} \exp \left[-\left(\varepsilon \tilde{\lambda}_{m}^{\varepsilon}+\frac{1}{4 \varepsilon}\right)(T-t)\right]\right\|_{L^{2}(\Omega, \mathrm{d} x)}
$$




$$
\leq C\left\|\varphi^{T}\right\|_{L^{2}(\Omega)} \exp \left(\frac{2 L+\delta-(T-t)}{4 \varepsilon}\right) .
$$

The proof of Lemma 5.1 is analogous to that of Lemma 3.3 since the only negative eigenvalue of (5.2) is $\tilde{\lambda}_{0}^{\varepsilon}$. In addition, we remark that, for all $m \in \mathbb{N}$ and $\bar{t} \in[t, T]$ :

$$
\begin{gathered}
\left(\int_{\Omega} \varphi^{T}(z) e^{(2 \varepsilon)^{-1} z} v_{m}^{\varepsilon}(z) \mathrm{d} z\right) v_{m}^{\varepsilon}(x) e^{-(2 \varepsilon)^{-1} x} \exp \left[-\left(\varepsilon \tilde{\lambda}_{m}^{\varepsilon}+\frac{1}{4 \varepsilon}\right)(T-t)\right] \\
=\left(\int_{\Omega} \varphi^{T}(z) e^{(2 \varepsilon)^{-1} z} v_{m}^{\varepsilon}(z) \mathrm{d} z\right) \exp \left[-\left(\varepsilon \tilde{\lambda}_{m}^{\varepsilon}+\frac{1}{4 \varepsilon}\right)(T-\bar{t})\right] v_{m}^{\varepsilon}(x) e^{-(2 \varepsilon)^{-1} x} \exp \left[-\left(\varepsilon \tilde{\lambda}_{m}^{\varepsilon}+\frac{1}{4 \varepsilon}\right)(\bar{t}-t)\right] \\
=\left(\int_{\Omega}\left\{\sum_{r \in \mathbb{N}}\left(\int_{\Omega} \varphi^{T}(z) e^{(2 \varepsilon)^{-1} z} v_{r}^{\varepsilon}(z) \mathrm{d} z\right) v_{r}^{\varepsilon}(\tilde{z}) e^{-(2 \varepsilon)^{-1} \tilde{z}} \exp \left[-\left(\varepsilon \tilde{\lambda}_{r}^{\varepsilon}+\frac{1}{4 \varepsilon}\right)(T-\bar{t})\right]\right\}\right. \\
\left.e^{(2 \varepsilon)^{-1} \tilde{z}} v_{m}^{\varepsilon}(\tilde{z}) d \tilde{z}\right) v_{m}^{\varepsilon}(x) e^{-(2 \varepsilon)^{-1} x} \exp \left[-\left(\varepsilon \tilde{\lambda}_{m}^{\varepsilon}+\frac{1}{4 \varepsilon}\right)(\bar{t}-t)\right] \\
=\left(\int_{\Omega} \varphi(\bar{t}, z) e^{(2 \varepsilon)^{-1} z} v_{m}^{\varepsilon}(z) \mathrm{d} z\right) v_{m}^{\varepsilon}(x) e^{-(2 \varepsilon)^{-1} x} \exp \left[-\left(\varepsilon \tilde{\lambda}_{m}^{\varepsilon}+\frac{1}{4 \varepsilon}\right)(\bar{t}-t)\right] .
\end{gathered}
$$

We have used in the second equality of (5.5) that the eigenfunctions of (5.2) form an orthonormal set of functions and Fubini's Theorem (it can be proved as in Lemma 3.3 that the series is convergent in $L^{2}(\Omega)$ ). A consequence of (5.5) and Lemma 5.1 is the following:

Corollary 5.2. Let $L>0$ and $\Omega:=(-L, 0)$. Then, for all $T_{0}>0$ and $\delta>0$ there is $C>0$ such that for all $\varphi^{T} \in L^{2}(\Omega), T \geq T_{0}, \bar{t} \in\left[T_{0}, T\right]$ and $t \in\left[0, \bar{t}-T_{0}\right]$ we have the estimate:

$$
\begin{aligned}
\| \sum_{m \in \mathbb{N}_{*}}\left(\int_{\Omega} \varphi^{T}(z) e^{(2 \varepsilon)^{-1} z} v_{m}(z) \mathrm{d} z\right) v_{m}(x) e^{-(2 \varepsilon)^{-1} x} \exp [ & \left.-\left(\varepsilon \tilde{\lambda}_{m}^{\varepsilon}+\frac{1}{4 \varepsilon}\right)(T-t)\right] \|_{L^{2}(\Omega, \mathrm{d} x)} \\
& \leq C\|\varphi(\bar{t}, \cdot)\|_{L^{2}(\Omega, \mathrm{d} x)} \exp \left(\frac{2 L+\delta-(\bar{t}-t)}{4 \varepsilon}\right) .
\end{aligned}
$$

In particular, we obtain that for all $T_{0}>0$ and $\delta>0$ there is $C>0$ such that for all $\varphi^{T} \in L^{2}(\Omega), T \geq T_{0}$ and $\bar{t} \in\left[T_{0}, T\right):$

$$
\begin{aligned}
\left\|\sum_{m \in \mathbb{N}_{*}}\left(\int_{\Omega} \varphi^{T}(z) e^{(2 \varepsilon)^{-1} z} v_{m}(z) \mathrm{d} z\right) v_{m}(x) e^{-(2 \varepsilon)^{-1} x} \exp \left[-\left(\varepsilon \tilde{\lambda}_{m}^{\varepsilon}+\frac{1}{4 \varepsilon}\right)(T-t)\right]\right\|_{L^{2}(\Omega, \mathrm{d} x)} \\
\leq \frac{C}{T-\bar{t}} \int_{\bar{t}}^{T}\|\varphi(s, \cdot)\|_{L^{2}(\Omega)} d s \exp \left(\frac{2 L+\delta-(\bar{t}-t)}{4 \varepsilon}\right) .
\end{aligned}
$$

End of the proof of Proposition 2.17. In order to prove Proposition 2.17 we split the solutions of system (4.2) into two parts with the help of Lemma 5.1. Indeed, we define:

$$
\varphi^{1}(x):=\frac{\left(\int_{\Omega} \varphi^{T}(z) \mathrm{d} z\right) e^{-\varepsilon x}}{\left\|e^{-(2 \varepsilon)^{-1} z}\right\|_{L^{2}(\Omega, \mathrm{d} z)}},
$$


and:

$$
\varphi^{2}(t, x):=\sum_{m \in \mathbb{N}_{*}}\left(\int_{\Omega} \varphi^{T}(z) e^{(2 \varepsilon)^{-1} z} v_{m}^{\varepsilon}(z) \mathrm{d} z\right) v_{m}^{\varepsilon}(x) e^{-(2 \varepsilon)^{-1} x} \exp \left[-\left(\varepsilon \tilde{\lambda}_{m}^{\varepsilon}+\frac{1}{4 \varepsilon}\right)(T-t)\right] .
$$

We remark that:

$$
\varphi(t, x)=\varphi^{1}(x)+\varphi^{2}(t, x), \text { in } Q .
$$

Next, we estimate $\varphi^{1}$ and $\varphi^{2}(0, \cdot)$ with respect to $\|\varphi\|_{L^{2}(Q)}$ :

Let us first estimate $\varphi^{1}$. If $\varphi^{1}=0$ the estimate is trivial. Consequently, we suppose from now on that $\varphi^{1} \neq 0$. We denote:

$$
\mathcal{N}:=\left\{t: \varphi^{2}(t, \cdot) \in B_{L^{2}(\Omega)}\left(-\varphi^{1}, \frac{\left\|\varphi^{1}\right\|_{L^{2}(\Omega)}}{2}\right)\right\}
$$

for:

$$
B_{X}(w, r):=\left\{v \in X:\|v-w\|_{X}<r\right\}
$$

We have that:

$$
|\mathcal{N}|<3 L
$$

since otherwise there are some $t_{1}$ and $t_{2}$ such that $t_{2}-t_{1} \geq 3 L$ and:

$$
\varphi^{2}\left(t_{1}, \cdot\right), \varphi^{2}\left(t_{2}, \cdot\right) \in B_{L^{2}(\Omega)}\left(-\varphi^{1}, \frac{\left\|\varphi^{1}\right\|_{L^{2}(\Omega)}}{2}\right)
$$

but by (5.4) this is impossible. Indeed, the system is autonomous and if $t_{2} \in \mathcal{N}$ we have the estimate:

$$
\left\|\varphi\left(t_{2}, \cdot\right)\right\|_{L^{2}(\Omega)} \leq \frac{\left\|\varphi^{1}\right\|_{L^{2}(\Omega)}}{2}
$$

so by (5.6) for $\bar{t}=t_{2}$ and $\delta=L / 2$ we have that $t_{1} \notin \mathcal{N}$ for $\varepsilon$ small enough. In addition, we remark that in $[0, T] \backslash \mathcal{N}$ we have that:

$$
\|\varphi(t, \cdot)\|_{L^{2}(\Omega)} \geq \frac{\left\|\varphi^{1}\right\|_{L^{2}(\Omega)}}{2}
$$

Thus, we obtain from (5.8) and (5.9) that for all $\varphi^{T} \in L^{2}(\Omega)$ and $T>4 L$ :

$$
\frac{\left\|\varphi^{1}\right\|_{L^{2}(\Omega)}}{\|\varphi\|_{L^{2}(Q)}} \leq \frac{\left\|\varphi^{1}\right\|_{L^{2}(\Omega)}}{\|\varphi\|_{L^{2}(((0, T) \backslash \mathcal{N}) \times \Omega)}} \leq \frac{2}{\sqrt{T-3 L}} \leq \frac{2}{\sqrt{T / 4}}=\frac{4}{\sqrt{T}}
$$

Let us now estimate $\varphi^{2}$. We have that:

$$
\frac{\left\|\varphi^{2}(0, \cdot)\right\|_{L^{2}(\Omega)}}{\|\varphi\|_{L^{2}(Q)}} \leq \frac{C e^{-c \varepsilon^{-1}}\|\varphi\|_{L^{2}((T-L, T) \times \Omega)}}{\|\varphi\|_{L^{2}(Q)}} \leq C e^{-c \varepsilon^{-1}},
$$


which is a direct consequence of (5.7) for $\bar{t}=T-L$. Thus, combining (5.10) and (5.11) we obtain (2.22) for $\varepsilon$ small enough.

\section{OPEN PROBLEMS}

We now present some issues that remain open:

- Study the controllability properties of (1.1) but with a non-constant speed; i.e. of (1.1) but with the first equation replaced by:

$$
y_{t}-\varepsilon \Delta y+\theta(t, x) \cdot \nabla y=1_{\omega} f
$$

for a function $\theta \in\left(L^{\infty}(Q)\right)^{d}$. The fact that there is no easy transformation like (2.3) to diagonalize the system, which we have used for both the decay property and the Carleman estimate, prevents to study this problem just with the techniques proposed in this paper. In addition, we cannot replicate the explicit computations of Section 4.

- Obtain precise estimates of the cost of the control problem (1.1) for vanishing diffusivity when $a^{\varepsilon}$ also depends on the time variable. Indeed, when $a^{\varepsilon}$ depends on the time variable, the eigenfunctions of (2.6) also depend on the time variable, which makes cumbersome working with the Fourier representation of the solutions.

- Determine if for all $\Omega, \omega \subset \Omega, T>0$ and $a^{\varepsilon} \in L^{\infty}(\Gamma)$ such that $\sup _{\varepsilon \in\left(0, \varepsilon_{0}\right)}\left\|a^{\varepsilon}\right\|_{L^{\infty}(\Gamma)}<\infty$ there is a constant $C>0$ depending on $T, \omega$ and $\Omega$ such that for $\varepsilon$ small enough we have that:

$$
K(\Omega, \omega, T, \varepsilon) \leq C e^{C \varepsilon^{-1}}
$$

Indeed, considering the equation (2.4) and adapting the proof of Proposition 3.5 we can easily obtain that:

$$
K(\Omega, \omega, T, \varepsilon) \leq C e^{C \varepsilon^{-4 / 3}} ;
$$

however, (6.1) would be more coherent with the literature (see [24]). The main difficulty to prove a Carleman inequality is absorbing the boundary terms.

- Determine for the control problems (2.12) and (2.16) and for all $y^{0} \in L^{2}(\Omega)$ whether the map $\varepsilon \mapsto f_{y^{0}}^{\varepsilon} \in$ $L^{2}(Q)$ is bounded in a neighbourhood of 0 , for $f_{y^{0}}^{\varepsilon}$ the optimal control (i.e. the control of smallest norm in $\left.L^{2}(Q)\right)$ such that $y(T, \cdot)=0$.

- Study the cost of the controllability in the context of (2.16), and in particular to determine if it explodes exponentially when $\varepsilon \rightarrow 0$. We know by Proposition 2.16 that this is false if $\omega=(-L, 0)$, but the other cases are still open. The main difficulty is that we do not obtain that the cost explodes just by computing the cost of observing the first eigenfunction. A similar open problem is to determine the cost of the null controllability of $(2.12)$ if $p_{l} \in \pi_{1}(\bar{\omega})$ (see (2.13) for the notation).

- Determine if Proposition 2.17 is still true if $\Omega$ is any arbitrary domain instead of a segment. The main new difficulty is that the exact value of $\tilde{\lambda}_{m}^{\varepsilon}$ is unknown.

Acknowledgements. I would like to thank my thesis advisor Sergio Guerrero and three anonymous referees for their multiple remarks. This work was partially supported by grants from Région Ile-de-France and by the ANR research project IFSMACS (ANR-15-CE40-0010). This project has received funding from the European Research Council (ERC) under the European Union's Horizon 2020 research and innovation programme (grant agreement NO. 694126-DyCon). 


\section{REFERENCES}

[1] H. Bahouri, J.-Y. Chemin and R. Danchin, Vol. 343 of Fourier analysis and nonlinear partial differential equations. Springer Science \& Business Media (2011).

[2] J.A. Bárcena-Petisco, Uniform controllability of a Stokes problem with a transport term in the zero-diffusion limit. SIAM J. Control Optim. 58 (2020) 1597-1625.

[3] J.A. Bárcena-Petisco, M. Cavalcante, G.M. Coclite, N. de Nitti and E. Zuazua, Control of hyperbolic and parabolic equations on networks and singular limits. Preprint hal-03233211 (2021).

[4] K. Bhandari and F. Boyer, Boundary null-controllability of coupled parabolic systems with Robin conditions. Evol. Equ. Control The. 10 (2021) 61-102.

[5] I.J. Bigio and S. Fantini, Quantitative biomedical optics: theory, methods, and applications. Cambridge University Press (2016).

[6] N. Carreño and S. Guerrero, On the non-uniform null controllability of a linear KdV equation. Asymptotic Anal. 94 (2015) 33-69.

[7] N. Carreño and S. Guerrero, Uniform null controllability of a linear KdV equation using two controls. J. Math. Anal. Appl. 457 (2018) 922-943.

[8] N. Carreño and P. Guzmán, On the cost of null controllability of a fourth-order parabolic equation. J. Differ. Equ. 261 (2016) 6485-6520.

[9] N. Carreño and C. Loyala, An explicit time for the uniform null controllability of a linear Korteweg-de Vries equation (2021).

[10] F.W. Chaves-Silva and G. Lebeau, Spectral inequality and optimal cost of controllability for the Stokes system. ESAIM: COCV 22 (2016) 1137-1162.

[11] P. Cornilleau and S. Guerrero, Controllability and observability of an artificial advection-diffusion problem. Math. Control Signal 24 (2012) 265-294.

[12] P. Cornilleau and S. Guerrero, On the cost of null-control of an artificial advection-diffusion problem. ESAIM: COCV 19 (2013) 1209-1224.

[13] J.-M. Coron, Control and Nonlinearity. Number 136. American Mathematical Soc. (2007).

[14] J.-M. Coron and S. Guerrero, Singular optimal control: a linear 1-D parabolic-hyperbolic example. Asymptotic Anal. 44 (2005) $237-257$.

[15] S. Ervedoza and E. Zuazua, Sharp observability estimates for heat equations. Arch. Ratl. Mech. An. 202 (2011) 975-1017.

[16] L.C. Evans, Partial Differential Equation. American Mathematical Society (2010), second edition.

[17] E. Fernández-Cara, M. González-Burgos, S. Guerrero and J.-P. Puel, Null controllability of the heat equation with boundary Fourier conditions: the linear case. ESAIM: COCV 12 (2006) 442-465.

[18] E. Fernández-Cara and S. Guerrero, Global carleman inequalities for parabolic systems and applications to controllability. SIAM J. Control. Optim. 45 (2006) 1395-1446.

[19] A.V. Fursikov and O. Yu. Imanuvilov, Controllability of evolution equations. Number 34. Seoul National University (1996).

[20] O. Glass, A complex-analytic approach to the problem of uniform controllability of a transport equation in the vanishing viscosity limit. J. Funct. Anal. 258 (2010) 852-868.

[21] O. Glass and S. Guerrero, On the uniform controllability of the Burgers equation. SIAM J. Control Optim. 46 (2007) $1211-1238$.

[22] O. Glass and S. Guerrero, Some exact controllability results for the linear KdV equation and uniform controllability in the zero-dispersion limit. Asymptotic Anal. 60 (2008) 61-100.

[23] O. Glass and S. Guerrero, Uniform controllability of a transport equation in zero diffusion-dispersion limit. Math. Mod. Meth. Appl. S. 19 (2009) 1567-1601.

[24] S. Guerrero and G. Lebeau, Singular optimal control for a transport-diffusion equation. Commun. Part. Diff. Eq. 32 (2007) $1813-1836$.

[25] V. Ivrii, 100 years of Weyl's law. B. Math. Sci. 6 (2016) 379-452.

[26] K. Kassab, Uniform controllability of a transport equation in zero fourth order equation-dispersion limit. Preprint hal-03080969 (2020).

[27] O.A. Ladyženskaja, V.A. Solonnikov and N.N. Ural'ceva, Vol. 23 of Linear and quasi-linear equations of parabolic type. American Mathematical Soc. (1988).

[28] C. Laurent and M. Léautaud, On uniform observability of gradient flows in the vanishing viscosity limit. J. de l'École polytechnique - Math. 8 (2021) 439-506.

[29] G. Lebeau and L. Robbiano, Contrôle exact de l'équation de la chaleur. Commun. Part. Diff. Eq. 20 (1995) $335-356$.

[30] J.-L. Lions, Contrôlabilité exacte, perturbations et stabilisation de systems distribués, tome 1, RMA 8 (1988).

[31] J.-L. Lions and E. Zuazua, A generique uniqueness result for the Stokes system and its control theoretical consequences. Part. Differ. Equ. Appl. 177 (1996) 221-235.

[32] P. Lissy, A link between the cost of fast controls for the 1-D heat equation and the uniform controllability of a 1-D transportdiffusion equation. C. R. Math. Acad. Sci. Paris 350 (2012) 591-595.

[33] P. Lissy, An application of a conjecture due to Ervedoza and Zuazua concerning the observability of the heat equation in small time to a conjecture due to Coron and Guerrero concerning the uniform controllability of a convection-diffusion equation in the vanishing viscosity limit. Syst. Control Lett. 69 (2014) 98-102.

[34] P. Lissy, Explicit lower bounds for the cost of fast controls for some 1-D parabolic or dispersive equations, and a new lower bound concerning the uniform controllability of the 1-D transport-diffusion equation. J. Differ. Equ. 259 (2015) 5331-5352. 
[35] M. López-García and A. Mercado, Uniform null controllability of a fourth-order parabolic equation with a transport term. J. Math. Anal. Appl. 498 (2021) 124979.

[36] D.L. Russell, Controllability and stabilizability theory for linear partial differential equations: recent progress and open questions. Siam Rev. 20 (1978) 639-739.

\section{Subscribe to Open (S20) A fair and sustainable open access model}

This journal is currently published in open access under a Subscribe-to-Open model (S2O). S2O is a transformative model that aims to move subscription journals to open access. Open access is the free, immediate, online availability of research articles combined with the rights to use these articles fully in the digital environment. We are thankful to our subscribers and sponsors for making it possible to publish this journal in open access, free of charge for authors.

\section{Please help to maintain this journal in open access!}

Check that your library subscribes to the journal, or make a personal donation to the $\mathrm{S} 2 \mathrm{O}$ programme, by contacting subscribers@edpsciences.org

More information, including a list of sponsors and a financial transparency report, available at: https://www.edpsciences.org/en/maths-s2o-programme 